\title{
VARIABLE DEPTH KDV EQUATIONS AND GENERALIZATIONS TO MORE NONLINEAR REGIMES
}

\author{
SAMER ISRAWI ${ }^{1}$
}

\begin{abstract}
We study here the water waves problem for uneven bottoms in a highly nonlinear regime where the small amplitude assumption of the Korteweg-de Vries (KdV) equation is enforced. It is known that, for such regimes, a generalization of the KdV equation (somehow linked to the CamassaHolm equation) can be derived and justified [Constantin and Lannes, Arch. Ration. Mech. Anal. 192 (2009) 165-186] when the bottom is flat. We generalize here this result with a new class of equations taking into account variable bottom topographies. Of course, many variable depth $\mathrm{KdV}$ equations existing in the literature are recovered as particular cases. Various regimes for the topography regimes are investigated and we prove consistency of these models, as well as a full justification for some of them. We also study the problem of wave breaking for our new variable depth and highly nonlinear generalizations of the $\mathrm{KdV}$ equations.
\end{abstract}

Mathematics Subject Classification. 35B40, 76B15.

Received January 14, 2009.

Published online January 27, 2010.

\section{INTRODUCTION}

\subsection{General setting}

This paper deals with the water waves problem for uneven bottoms, which consists in studying the motion of the free surface and the evolution of the velocity field of a layer of fluid under the following assumptions: the fluid is ideal, incompressible, irrotational, and under the only influence of gravity. Earlier works have set a good theoretical background for this problem. Its well-posedness has been discussed among others by Nalimov [27], Yosihara [37], Craig [9], $\mathrm{Wu}[34,35]$ and Lannes [23]. Nevertheless, the solutions of these equations are very difficult to describe, because of the complexity of these equations. At this point, a classical method is to choose an asymptotic regime, in which we look for approximate models and hence for approximate solutions. More recently Alvarez-Samaniego and Lannes [2] rigorously justified the relevance of the main asymptotical models used in coastal oceanography, including: shallow-water equations, Boussinesq systems, Kadomtsev-Petviashvili (KP) approximation, Green-Naghdi equations (GN), Serre approximation, full-dispersion model and deep-water equations. Some of these models capture the existence of solitary water waves and the associated phenomenon of soliton manifestation [17]. The most prominent example is the Korteweg-de Vries (KdV) equation [21], that is

\footnotetext{
Keywords and phrases. Water waves, KdV equations, topographic effects.

${ }^{1}$ Laboratoire de Mathématiques Appliquées de Bordeaux, Université Bordeaux 1, 351 Cours de la Libération, 33405 Talence Cedex, France. Samer.Israwi@math.u-bordeaux1.fr
} 
integrable and relevant for the phenomenon of soliton manifestation. The KdV approximation originally derived over flat bottoms has been rigorously justified in $[6,9,16,31]$. When the bottom is not flat, various generalizations of the KdV equation with non constant coefficients have been proposed $[12,15,19,20,26,29,30,32,33,36]$. One of the aims of this article is to justify the derivation of this Korteweg-de Vries equation with topography (called KdV-top). Another development of models for water waves was initiated in order to gain insight into wave breaking, one of the most fundamental aspects of water waves [13]. In 2008 Constantin and Lannes [8] rigorously justified the relevance of more nonlinear generalization of the KdV equations (linked to the CamassaHolm equation [7] and the Degasperis-Procesi equations [11]) as models for the propagation of shallow water waves. They proved that these equations can be used to furnish approximations to the governing equations for water waves, and in their investigation they put earlier (formal) asymptotic procedures due to Johnson [18] on a firm and mathematically rigorous basis. However, all these results hold for flat bottoms only. The main goal of this article is to investigate the same scaling as in [8] and to include topographical effects. To this end, we derive a new variable coefficients class of equations which takes into account these effects and generalizes the $\mathrm{CH}$ like equations of Constantin-Lannes [8]. The presence of the topography terms induce secular growth effects which do not always allow a full justification of the model. We however give some consistency results for all the models derived here, and then show that under some additional assumptions on the topography variations, the secular terms can be controlled and a full justification given.

\subsection{Presentation of the results}

Parameterizing the free surface by $z=\zeta(t, x)$ (with $x \in \mathbb{R}$ ) and the bottom by $z=-h_{0}+b(x)$ (with $h_{0}>0$ constant), one can use the incompressibility and irrotationality conditions to write the water-waves equations under Bernoulli's formulation, in terms of a velocity potential $\varphi$ associated with the flow, and where $\varphi(t,$.$) is$ defined on $\Omega_{t}=\left\{(x, z),-h_{0}+b(x)<z<\zeta(t, x)\right\}$ (i.e. the velocity field is given by $v=\nabla_{x, z} \varphi$ ):

$$
\begin{cases}\partial_{x}^{2} \varphi+\partial_{z}^{2} \varphi=0, & \text { in } \Omega_{t}, \\ \partial_{n} \varphi=0, & \text { at } z=-h_{0}+b \\ \partial_{t} \zeta+\partial_{x} \zeta \partial_{x} \varphi=\partial_{z} \varphi, & \text { at } z=\zeta, \\ \partial_{t} \varphi+\frac{1}{2}\left(\left(\partial_{x} \varphi\right)^{2}+\left(\partial_{z} \varphi\right)^{2}\right)+g \zeta=0, & \text { at } z=\zeta,\end{cases}
$$

where $g$ is the gravitational acceleration, $\partial_{n} \varphi$ is the outward normal derivative at the boundary of the fluid domain. The qualitative study of the water-waves equations is made easier by the introduction of dimensionless variables and unknowns. This requires the introduction of various orders of magnitude linked to the physical regime under consideration. More precisely, let us introduce the following quantities: $a$ is a typical amplitude of the waves; $\lambda$ is the wave-length of the waves; $b_{0}$ is the order of amplitude of the variations of the bottom topography; $\lambda / \alpha$ is the wave-length of the bottom variations; $h_{0}$ is the reference depth. We also introduce the following dimensionless parameters:

$$
\varepsilon=\frac{a}{h_{0}}, \quad \mu=\frac{h_{0}^{2}}{\lambda^{2}}, \quad \beta=\frac{b_{0}}{h_{0}}
$$

the parameter $\varepsilon$ is often called nonlinearity parameter; while $\mu$ is the shallowness parameter. We now perform the classical shallow water non-dimensionalization using the following relations:

$$
\begin{aligned}
& x=\lambda x^{\prime}, \quad z=h_{0} z^{\prime}, \quad \zeta=a \zeta^{\prime}, \\
& \varphi=\frac{a}{h_{0}} \lambda \sqrt{g h_{0}} \varphi^{\prime}, \quad b=b_{0} b^{\prime}, \quad t=\frac{\lambda}{\sqrt{g h_{0}}} t^{\prime} ;
\end{aligned}
$$


so, the equations of motion (1.1) then become (after dropping the primes for the sake of clarity):

$$
\begin{cases}\mu \partial_{x}^{2} \varphi+\partial_{z}^{2} \varphi=0, & \text { at }-1+\beta b^{(\alpha)}<z<\varepsilon \zeta \\ \partial_{z} \varphi-\mu \beta \alpha \partial_{x} b^{(\alpha)} \partial_{x} \varphi=0, & \text { at } z=-1+\beta b^{(\alpha)}, \\ \partial_{t} \zeta-\frac{1}{\mu}\left(\mu \varepsilon \partial_{x} \zeta \partial_{x} \varphi+\partial_{z} \varphi\right)=0, & \text { at } z=\varepsilon \zeta, \\ \partial_{t} \varphi+\frac{1}{2}\left(\varepsilon\left(\partial_{x} \varphi\right)^{2}+\frac{\varepsilon}{\mu}\left(\partial_{z} \varphi\right)^{2}\right)+\zeta=0, & \text { at } z=\varepsilon \zeta\end{cases}
$$

where $b^{(\alpha)}(x)=b(\alpha x)$. Making assumptions on the size of $\varepsilon, \beta$, $\alpha$, and $\mu$ one is led to derive (simpler) asymptotic models from (1.3). In the shallow-water scaling $(\mu \ll 1)$, one can derive (when no smallness assumption is made on $\varepsilon, \beta$ and $\alpha$ ) the so-called Green-Naghdi equations (see [14,25] for a derivation and [2] for a rigorous justification). For one-dimensional surfaces and over uneven bottoms these equations couple the free surface elevation $\zeta$ to the vertically averaged horizontal component of the velocity,

$$
u(t, x)=\frac{1}{1+\varepsilon \zeta-\beta b^{(\alpha)}} \int_{-1+\beta b^{(\alpha)}}^{\varepsilon \zeta} \partial_{x} \varphi(t, x, z) \mathrm{d} z
$$

and can be written as:

$$
\left\{\begin{array}{l}
\partial_{t} \zeta+\partial_{x}(h u)=0 \\
\left(1+\frac{\mu}{h} \mathcal{T}\left[h, \beta b^{(\alpha)}\right]\right) \partial_{t} u+\partial_{x} \zeta+\varepsilon u \partial_{x} u \\
\quad+\mu \varepsilon\left\{-\frac{1}{3 h} \partial_{x}\left(h^{3}\left(u \partial_{x}^{2} u\right)-\left(\partial_{x} u\right)^{2}\right)+\Im\left[h, \beta b^{(\alpha)}\right] u\right\}=0
\end{array}\right.
$$

where $h=1+\varepsilon \zeta-\beta b^{(\alpha)}$ and

$$
\mathcal{T}\left[h, \beta b^{(\alpha)}\right] W=-\frac{1}{3} \partial_{x}\left(h^{3} \partial_{x} W\right)+\frac{\beta}{2} \partial_{x}\left(h^{2} \partial_{x} b^{(\alpha)}\right) W+\beta^{2} h\left(\partial_{x} b^{(\alpha)}\right)^{2} W
$$

while the purely topographical term $\Im\left[h, \beta b^{(\alpha)}\right] u$ is defined as:

$$
\Im\left[h, \beta b^{(\alpha)}\right] u=\frac{\beta}{2 h}\left[\partial_{x}\left(h^{2}\left(u \partial_{x}\right)^{2} b^{(\alpha)}\right)-h^{2}\left(\left(u \partial_{x}^{2} u\right)-\left(\partial_{x} u\right)^{2}\right) \partial_{x} b^{(\alpha)}\right]+\beta^{2}\left(\left(u \partial_{x}\right)^{2} b^{(\alpha)}\right) \partial_{x} b^{(\alpha)} .
$$

If we make the additional assumption that $\varepsilon \ll 1, \beta \ll 1$ then the above system reduces at first order to a wave equation of speed \pm 1 and any perturbation of the surface splits up into two components moving in opposite directions. A natural issue is therefore to describe more accurately the motion of these two "unidirectional" waves. In the so called long-wave regime

$$
\mu \ll 1, \quad \varepsilon=O(\mu),
$$

and for flat bottoms, Korteweg and de Vries [21] found that say, the right-going wave should satisfy the KdV equation:

$$
u_{t}+u_{x}+\frac{3}{2} \varepsilon u u_{x}+\frac{\mu}{6} u_{x x x}=0
$$

and $(\zeta=u+O(\varepsilon, \mu))$.

At leading order, this equation reduces to the expected transport equation at speed 1 . It has been noticed by Benjamin et al. [4] that the KdV equation belongs to a wider class of equations. For instance, the BBM equation first used by Peregrine [28], and sometimes also called the regularized long-wave equation, provides 
an approximation of the exact water waves equations of the same accuracy as the KdV equation and can be written under the form ${ }^{2}$ :

$$
u_{t}+u_{x}+\frac{3}{2} \varepsilon u u_{x}+\mu\left(A u_{x x x}+B u_{x x t}\right)=0 \quad \text { with } A-B=\frac{1}{6} .
$$

For higher values of $\varepsilon$, the nonlinear effects are stronger; in the regime

$$
\mu \ll 1, \quad \varepsilon=O(\sqrt{\mu}),
$$

the BBM equations (1.8) should be replaced by the following family (see $[8,18])$ :

$$
u_{t}+u_{x}+\frac{3}{2} \varepsilon u u_{x}+\mu\left(A u_{x x x}+B u_{x x t}\right)=\varepsilon \mu\left(E u u_{x x x}+F u_{x} u_{x x}\right),
$$

(with some conditions on $A, B, E$, and $F$ ) in order to keep the same $O\left(\mu^{2}\right)$ accuracy of the approximation. However, all these results only hold for flat bottoms; for the situation of an uneven bottom, various generalizations of the KdV equations with non constant coefficients have been proposed [12,15,19,20,26,29,30,32,33,36]. We justify in this paper the derivation of the generalized KdV equation and also we show that the correct generalization of equation (1.10) under the scaling (1.9) and with the following conditions on the topographical variations:

$$
\beta \alpha=O(\mu), \quad \beta \alpha^{2}=O\left(\mu^{2}\right), \quad \beta \alpha \varepsilon=O\left(\mu^{2}\right),
$$

is given by:

$$
u_{t}+c u_{x}+\frac{3}{2} c_{x} u+\frac{3}{2} \varepsilon u u_{x}+\mu\left(\tilde{A} u_{x x x}+B u_{x x t}\right)=\varepsilon \mu \tilde{E} u u_{x x x}+\varepsilon \mu\left(\partial_{x}\left(\frac{\tilde{F}}{2} u\right) u_{x x}+u_{x} \partial_{x}^{2}\left(\frac{\tilde{F}}{2} u\right)\right),
$$

where $c=\sqrt{1-\beta b^{(\alpha)}}$ and $\tilde{A}, \tilde{E}, \tilde{F}$ differ from the coefficients $A, E, F$ in (1.10) because of topographic effects:

$$
\begin{aligned}
\tilde{A} & =A c^{5}-B c^{5}+B c, \\
\tilde{E} & =E c^{4}-\frac{3}{2} B c^{4}+\frac{3}{2} B, \\
\tilde{F} & =F c^{4}-\frac{9}{2} B c^{4}+\frac{9}{2} B .
\end{aligned}
$$

Notice that for an equation of the family (1.12) to be linearly well-posed it is necessary that $B \leq 0$. In Section 2 , we derive asymptotical approximations of the Green-Naghdi equations over non flat bottoms: equations on the velocity are given in Section 2.1 and equations on the surface elevation are obtained in Section 2.2; for these equations, $L^{\infty}$-consistency results are given (see Def. 2.1). In Section 2.3, the same kind of result is given in the (more restrictive) KdV scaling in order to recover the many variable depth KdV equations formally derived by oceanographers. Section 3 is devoted to the study of the well posedness of the equations derived in Section 2. Two different approaches are used, depending on the coefficient $B$ in (1.12): Section 3.1 deals with the case $B<0$ (in that case, further investigation on the breaking of waves can be performed, see Sect. 3.2) and Section 3.3 treats the case $B=0$. While secular growth effects prevent is from proving $H^{s}$-consistency (see Def. 4.1) for the models derived in Section 2, we show in Section 4 that such results hold if one makes stronger assumptions on the parameters. A full justification of the models can then be given (see Thm. 4.2).

\footnotetext{
${ }^{2}$ The BBM equation corresponds to $A=0$ in (1.8), and $\mathrm{KdV}$ to $B=0$. The intermediate cases can be found in [5].
} 


\section{Unidirectional Limit of the GREEN-NAGHDi EQUATIONS OVER UNEVEN Bottom IN THE CH AND KDV SCALINGS}

We derive here asymptotical approximations of the Green-Naghdi equations over non flat bottoms in the scalings (1.11) and (1.9). We remark that the Green-Naghdi equations can then be simplified into (denoting $\left.h=1+\varepsilon \zeta-\beta b^{(\alpha)}\right)$ :

$$
\left\{\begin{array}{l}
\zeta_{t}+[h u]_{x}=0 \\
u_{t}+\zeta_{x}+\varepsilon u u_{x}=\frac{\mu}{3 h}\left[h^{3}\left(u_{x t}+\varepsilon u u_{x x}-\varepsilon u_{x}^{2}\right)\right]_{x}
\end{array}\right.
$$

where $O\left(\mu^{2}\right)$ terms have been discarded.

We consider here parameters $\varepsilon, \beta, \alpha$ and $\mu$ linked by the relations

$$
\varepsilon=O(\sqrt{\mu}), \beta \alpha=O(\varepsilon), \beta \alpha=O(\mu), \beta \alpha^{2}=O\left(\mu^{2}\right), \beta \alpha \varepsilon=O\left(\mu^{2}\right)
$$

(note that in the case of flat bottoms, one can take $\beta=0$, so that this set of relations reduce to $\varepsilon=O(\sqrt{\mu})$ ).

Equations for the velocity $u$ are first derived in Section 2.1 and equations for the surface elevation $\zeta$ are obtained in Section 2.2. The considerations we make on the derivation of these equations are related to the approach initiated by Constantin and Lannes [8]. In addition, in Section 2.3 we recover and justify the KdV equation over a slowly varying depth (formally derived in $[12,20,32]$ ).

\subsection{Equations on the velocity}

If we want to find an approximation at order $O\left(\mu^{2}\right)$ of the GN equations under the scalings (2.2), it is natural to look for $u$ as a solution of (1.12) with variable coefficients $\widetilde{A}, \widetilde{B}, \widetilde{E}, \widetilde{F}$ to be determined. We prove in this section that one can associate to the solution of (1.12) a family of approximate solutions consistent with the Green-Naghdi equations (2.1) in the following sense:

Definition 2.1. Let $\wp$ be a family of parameters $\theta=(\varepsilon, \beta, \alpha, \mu)$ satisfying (2.2). A family $\left(\zeta^{\theta}, u^{\theta}\right)_{\theta \in \wp}$ is $L^{\infty}$-consistent on $\left[0, \frac{T}{\varepsilon}\right]$ with the GN equations (2.1), if for all $\theta \in \wp\left(\right.$ and denoting $\left.h^{\theta}=1+\varepsilon \zeta^{\theta}-\beta b^{(\alpha)}\right)$,

$$
\left\{\begin{array}{l}
\zeta_{t}^{\theta}+\left[h^{\theta} u^{\theta}\right]_{x}=\mu^{2} r_{1}^{\theta} \\
u_{t}^{\theta}+\zeta_{x}^{\theta}+\varepsilon u^{\theta} u_{x}^{\theta}=\frac{\mu}{3 h^{\theta}}\left[\left(h^{\theta}\right)^{3}\left(u_{x t}^{\theta}+\varepsilon u^{\theta} u_{x x}^{\theta}-\varepsilon\left(u_{x}^{\theta}\right)^{2}\right)\right]_{x}+\mu^{2} r_{2}^{\theta}
\end{array}\right.
$$

with $\left(r_{1}^{\theta}, r_{2}^{\theta}\right)_{\theta \in \wp}$ bounded in $L^{\infty}\left(\left[0, \frac{T}{\varepsilon}\right] \times \mathbb{R}\right)$.

Remark 2.1. The notion of $L^{\infty}$-consistency is weaker then the notion of $H^{s}$-consistency given in Section 4 (Def. 4.1) and does not allow a full justification of the asymptotic models. Since secular growth effects do not allow in general an $H^{s}$-consistency, we state here an $L^{\infty}$-consistency result under very general assumptions on the topography parameters $\alpha$ and $\beta$. $H^{s}$-consistency and full justification of the models will then be achieved under additional assumptions in Section 4.

The following proposition shows that there is a one parameter family of equations $(1.12) L^{\infty}$-consistent with the GN equations (2.1). (For the sake of simplicity, here and throughout the rest of this paper, we take an infinitely smooth bottom parameterized by the function $b$.) 
Proposition 2.1. Let $b \in H^{\infty}(\mathbb{R})$ and $p \in \mathbb{R}$. Assume that

$$
A=p, \quad B=p-\frac{1}{6}, \quad E=-\frac{3}{2} p-\frac{1}{6}, \quad F=-\frac{9}{2} p-\frac{23}{24} .
$$

Then:

- For any family $\wp$ of parameters satisfying (2.2),

- For all $s \geq 0$ large enough and $T>0$,

- For any bounded family $\left(u^{\theta}\right)_{\theta \in \wp} \in C\left(\left[0, \frac{T}{\varepsilon}\right], H^{s}(\mathbb{R})\right)$ solving (1.12), the family $\left(\zeta^{\theta}, u^{\theta}\right)_{\theta \in \wp}$ with (omitting the index $\theta$ )

$$
\zeta:=c u+\frac{1}{2} \int_{-\infty}^{x} c_{x} u+\frac{\varepsilon}{4} u^{2}+\frac{\mu}{6} c^{4} u_{x t}-\varepsilon \mu c^{4}\left[\frac{1}{6} u u_{x x}+\frac{5}{48} u_{x}^{2}\right],
$$

is $L^{\infty}$-consistent on $\left[0, \frac{T}{\varepsilon}\right]$ with the $G N$ equations (2.1).

Remark 2.2. If we take $b=0$ - i.e. if we consider a flat bottom - then one can recover the equation (7) of [8] and equations (26a) and (26b) of [18] with $p=-\frac{1}{12}$ and $p=\frac{1}{6}$ respectively.

Proof. For the sake of simplicity, we denote by $O(\mu)$ any family of functions $\left(f^{\theta}\right)_{\theta \in \wp}$ such that $\frac{1}{\mu} f^{\theta}$ remains bounded in $L^{\infty}\left(\left[0, \frac{T}{\varepsilon}\right], H^{r}(\mathbb{R})\right)$ for all $\theta \in \wp$ (and for possibly different values of $r$ ). The same notation is also used for real numbers, e.g. $\varepsilon=O(\mu)$, but this should not yield any confusion. We use the notation $O_{L^{\infty}}(\mu)$ if $\frac{1}{\mu} f^{\theta}$ remains bounded in $L^{\infty}\left(\left[0, \frac{T}{\varepsilon}\right] \times \mathbb{R}\right)$. Of course, similar notations are used for $O\left(\mu^{2}\right)$ etc. To simplify the text, we also omit the index $\theta$ and write $u$ instead of $u^{\theta}$.

Step 1. We begin the proof by the following lemma where a new class of equations is deduced from (1.12). The coefficients $A, B, E, F$ in this new class of equations are constants (as opposed to $\widetilde{A}, \widetilde{E}$ and $\widetilde{F}$ in (1.12) that are functions of $x$ ).

Lemma 2.1. Under the assumptions of Proposition 2.1, there is a family $\left(R^{\theta}\right)_{\theta \in \wp}$ bounded in $L^{\infty}\left(\left[0, \frac{T}{\varepsilon}\right], H^{r}(\mathbb{R})\right)$ (for some $r<s$ ) such that (omitting the index $\theta$ )

$u_{t}+c u_{x}+\frac{3}{2} c_{x} u+\frac{3}{2} \varepsilon u u_{x}+\mu c^{5} A u_{x x x}+\mu B \partial_{x}\left(c^{4} u_{x t}\right)=\varepsilon \mu c^{4} E u u_{x x x}+\varepsilon \mu \frac{1}{2} F\left(c^{4} u\right)_{x} u_{x x}+\varepsilon \mu \frac{1}{2} F u_{x}\left(c^{4} u\right)_{x x}+\mu^{2} R$.

Proof. Remark that the relation $\alpha \beta=O(\mu)$ and the definitions of $\tilde{A}, \tilde{B}$, and $\tilde{E}$ in terms of $A, B, E$ and $F$ imply that

$$
\begin{aligned}
\mu\left(-B c^{5}+B c\right) u_{x x x}+\varepsilon \mu\left(-\frac{3}{2} B c^{4}+\frac{3}{2} B\right) u u_{x x x} & +\varepsilon \mu \partial_{x}\left(\left(\frac{-\frac{9}{2} B c^{4}+\frac{9}{2} B}{2}\right) u\right) u_{x x}+\varepsilon \mu u_{x} \partial_{x}^{2}\left(\left(\frac{-\frac{9}{2} B c^{4}+\frac{9}{2} B}{2}\right) u\right) \\
& =-\mu B \partial_{x}\left(c\left(c^{4}-1\right) \partial_{x} u_{x}\right)-\frac{3}{2} \mu \varepsilon B \partial_{x}\left(\left(c^{4}-1\right) \partial_{x}\left(u u_{x}\right)\right) \\
& =-\mu B \partial_{x}\left[\left(c^{4}-1\right) \partial_{x}\left(c u_{x}+\frac{3}{2} \varepsilon u u_{x}\right)\right]+O\left(\mu^{2}\right) \\
& =\mu B \partial_{x}\left(\left(c^{4}-1\right) u_{x t}\right)+O\left(\mu^{2}\right),
\end{aligned}
$$

the last line being a consequence of the identity $u_{t}=-\left(c u_{x}+\frac{3}{2} \varepsilon u u_{x}\right)+O(\mu)$ provided by (1.12) since we have

$$
\left|c_{x} u\right|_{H^{r}}=\left|-\frac{1}{2 c} \beta \alpha b_{x}^{(\alpha)} u\right|_{H^{r}} \leq \operatorname{Cst} \alpha \beta\left|\partial_{x} b^{(\alpha)}\right|_{W^{[r]+1, \infty}}|u|_{H^{r}}=O(\beta \alpha)=O(\mu)
$$


where $[r]$ is the largest integer smaller or equal to $r$. Equation (1.12) can thus be written under the form:

$u_{t}+c u_{x}+\frac{3}{2} c_{x} u+\frac{3}{2} \varepsilon u u_{x}+\mu c^{5} A u_{x x x}+\mu B \partial_{x}\left(c^{4} u_{x t}\right)=\varepsilon \mu c^{4} E u u_{x x x}+\varepsilon \mu \frac{1}{2} F\left(c^{4} u\right)_{x} u_{x x}+\varepsilon \mu \frac{1}{2} F u_{x}\left(c^{4} u\right)_{x x}+O\left(\mu^{2}\right)$,

which is exactly the result stated in the lemma.

If $u$ satisfies (1.12) one also has

$$
\begin{aligned}
u_{t}+c u_{x}+\frac{3}{2} \varepsilon u u_{x} & =-\frac{3}{2} c_{x} u+O(\mu) \\
& =O(\mu) .
\end{aligned}
$$

Differentiating (2.5) twice with respect to $x$, and using again the fact that $c_{x} f=O(\beta \alpha)=O(\mu)$ for all $f$ smooth enough, one gets

$$
c u_{x x x}=-u_{x x t}-\frac{3}{2} \varepsilon \partial_{x}^{2}\left(u u_{x}\right)+O(\mu) .
$$

It is then easy to deduce that

$$
\begin{aligned}
c^{5} u_{x x x} & =-c^{4} u_{x x t}-\frac{3}{2} c^{4} \varepsilon \partial_{x}^{2}\left(u u_{x}\right)+O(\mu) \\
& =-\partial_{x}\left(c^{4} u_{x t}\right)-\frac{3}{2} \varepsilon c^{4}\left(u u_{x x x}+3 u_{x} u_{x x}\right)+O(\mu)
\end{aligned}
$$

so that we can replace the $c^{5} u_{x x x}$ term of (2.4) by this expression. By using Lemma 2.1, one gets therefore the following equation where the linear term in $u_{x x x}$ has been removed:

$$
u_{t}+c u_{x}+\frac{3}{2} c_{x} u+\frac{3}{2} \varepsilon u u_{x}+\mu c^{4} a u_{x x t}=\varepsilon \mu c^{4}\left[e u u_{x x}+d u_{x}^{2}\right]_{x}+O\left(\mu^{2}\right)
$$

with $a=B-A, e=E+\frac{3}{2} A, d=\frac{1}{2}(F+3 A-E)$.

Step 2. We seek $v$ such that if $\zeta:=c u+\varepsilon v$ and $u$ solves (1.12) then the second equation of (2.1) is satisfied up to a $O\left(\mu^{2}\right)$ term. This is equivalent to checking that

$$
\begin{aligned}
u_{t}+[c u+\varepsilon v]_{x}+\varepsilon u u_{x}= & \mu\left(1-\beta b^{(\alpha)}\right) \varepsilon \partial_{x}(c u) u_{x t}+\frac{\mu}{3}\left(\left(1-\beta b^{(\alpha)}\right)+\varepsilon c u\right)^{2} u_{x x t} \\
& +\frac{\varepsilon \mu}{3}\left(1-\beta b^{(\alpha)}\right)^{2}\left(u u_{x x}-u_{x}^{2}\right)_{x}+O\left(\mu^{2}\right) \\
= & \frac{\mu}{3} c^{4} u_{x x t}+\varepsilon \mu\left(c^{3} u_{x} u_{x t}+\frac{2}{3} c^{3} u u_{x x t}+\frac{c^{4}}{3}\left(u u_{x x}-u_{x}^{2}\right)_{x}\right) \\
& +O\left(\mu^{2}\right),
\end{aligned}
$$

where we used the relations $O\left(\varepsilon^{2}\right)=O(\mu), O(\beta \alpha)=O(\mu)$ and the fact that $c^{2}=1-\beta b^{(\alpha)}$. This condition can be recast under the form

$$
\begin{aligned}
& \varepsilon v_{x}+\left[u_{t}+c u_{x}+\frac{3}{2} c_{x} u+\frac{3}{2} \varepsilon u u_{x}+\mu c^{4} a u_{x x t}-\varepsilon \mu c^{4}\left[e u u_{x x}+d u_{x}^{2}\right]_{x}\right] \\
= & \frac{1}{2} c_{x} u+\frac{\varepsilon}{2} u u_{x}+\mu c^{4}\left(a+\frac{1}{3}\right) u_{x x t}+\varepsilon \mu c^{3}\left(u_{x} u_{x t}+\frac{2}{3} u u_{x x t}+c\left[\left(\frac{1}{3}-e\right) u u_{x x}-\left(\frac{1}{3}+\mathrm{d}\right) u_{x}^{2}\right]_{x}\right)+O\left(\mu^{2}\right) .
\end{aligned}
$$


Since moreover one gets from (2.5) that $u_{x t}=-c u_{x x}+O(\mu, \varepsilon)$ and $u_{x x t}=-c u_{x x x}+O(\mu, \varepsilon)$, one gets readily

$$
\begin{aligned}
\varepsilon v_{x}+\left[u_{t}+c u_{x}+\frac{3}{2} c_{x} u\right. & \left.+\frac{3}{2} \varepsilon u u_{x}+\mu c^{4} a u_{x x t}-\varepsilon \mu\left[e u u_{x x}+d u_{x}^{2}\right]_{x}\right] \\
= & \frac{1}{2} c_{x} u+\frac{\varepsilon}{2} u u_{x}+\mu c^{4}\left(a+\frac{1}{3}\right) u_{x x t}-\varepsilon \mu c^{4}\left[\left(e+\frac{1}{3}\right) u u_{x x}+\left(d+\frac{1}{2}\right) u_{x}^{2}\right]_{x}+O\left(\mu^{2}\right) .
\end{aligned}
$$

From Step 1, we know that the term between brackets in the l.h.s. of this equation is of order $O\left(\mu^{2}\right)$ so that the second equation of (2.1) is satisfied up to $O\left(\mu^{2}\right)$ terms if

$$
\varepsilon v_{x}=\frac{1}{2} c_{x} u+\frac{\varepsilon}{2} u u_{x}+\mu c^{4}\left(a+\frac{1}{3}\right) u_{x x t}-\varepsilon \mu c^{4}\left[\left(e+\frac{1}{3}\right) u u_{x x}+\left(d+\frac{1}{2}\right) u_{x}^{2}\right]_{x}+O\left(\mu^{2}\right) .
$$

At this point we need also the following lemma.

Lemma 2.2. With $u$ and $b$ as in the statement of Proposition 2.1 , the mapping $(t, x) \longrightarrow \int_{-\infty}^{x} c_{x} u \mathrm{~d} x$ is well defined on $\left[0, \frac{T}{\varepsilon}\right] \times \mathbb{R}$.

Moreover one has that

$$
\left|\int_{-\infty}^{x} c_{x} u \mathrm{~d} x\right|_{L^{\infty}\left(\left[0, \frac{T}{\varepsilon}\right] \times \mathbb{R}\right)} \leq \operatorname{Cst} \sqrt{\alpha} \beta\left|b_{x}\right|_{2}|u|_{2}
$$

Proof. We used here the Cauchy-Schwarz inequality and the definition $c^{2}=1-\beta b^{(\alpha)}$ to get

$$
\int_{-\infty}^{x} c_{x} u \mathrm{~d} x \leq \operatorname{Cst} \alpha \beta\left|\left(b_{x}\right)^{(\alpha)}\right|_{2}|u|_{2} \leq \operatorname{Cst} \sqrt{\alpha} \beta\left|b_{x}\right|_{2}|u|_{2}<\infty .
$$

It is then easy to conclude the proof of the lemma.

Thanks to this lemma there is a solution $v \in C\left(\left[0, \frac{T}{\varepsilon}\right] \times \mathbb{R}\right)$ to $(2.7)$, namely

$$
\varepsilon v=\frac{1}{2} \int_{-\infty}^{x} c_{x} u+\frac{\varepsilon}{4} u^{2}+\mu c^{4}\left(a+\frac{1}{3}\right) u_{x t}-\varepsilon \mu c^{4}\left[\left(\mathrm{e}+\frac{1}{3}\right) u u_{x x}+\left(d+\frac{1}{2}\right) u_{x}^{2}\right] .
$$

Step 3. We show here that it is possible to choose the coefficients $A, B, E, F$ such that the first equation of (2.1) is also satisfied up to $O_{L^{\infty}}\left(\mu^{2}\right)$ terms. This is equivalent to checking that

$$
[c u+\varepsilon v]_{t}+\left[\left(1+\varepsilon(c u+\varepsilon v)-\beta b^{(\alpha)}\right) u\right]_{x}=0 .
$$

Remarking that the relations $O(\beta \alpha)=O(\mu), O\left(\beta \alpha^{2}\right)=O\left(\mu^{2}\right)$, and $O(\beta \alpha \varepsilon)=O\left(\mu^{2}\right)$ imply that

$$
\frac{1}{2} \int_{-\infty}^{x} c_{x} u_{t}=-\frac{1}{2} c c_{x} u+O_{L^{\infty}}\left(\mu^{2}\right)
$$


one infers from (2.8) that

$$
\begin{aligned}
\varepsilon v_{t}= & \frac{1}{2} \int_{-\infty}^{x} c_{x} u_{t}+\frac{\varepsilon}{2} u u_{t}+\mu c^{4}\left(a+\frac{1}{3}\right) u_{x t t}-\varepsilon \mu c^{4}\left[\left(e+\frac{1}{3}\right) u u_{x x}+\left(d+\frac{1}{2}\right) u_{x}^{2}\right]_{t} \\
= & -\frac{1}{2} c c_{x} u-\frac{\varepsilon}{2} u\left(c u_{x}+\frac{3 \varepsilon}{2} u u_{x}+\mu c^{4} a u_{x x t}\right)-\mu c^{4}\left(a+\frac{1}{3}\right) \partial_{x t}^{2}\left(c u_{x}+\varepsilon \frac{3}{2} u u_{x}\right) \\
& +\varepsilon \mu c^{5}\left[\left(e+\frac{1}{3}\right) u u_{x x}+\left(d+\frac{1}{2}\right) u_{x}^{2}\right]_{x}+O\left(\mu^{2}\right)+O_{L^{\infty}}\left(\mu^{2}\right) \\
= & -\frac{1}{2} c c_{x} u-\varepsilon \frac{1}{2} c u u_{x}-\varepsilon^{2} \frac{3}{4} u^{2} u_{x}-\mu\left(a+\frac{1}{3}\right) c^{5} u_{x x t} \\
& +\varepsilon \mu c^{5}\left[\left(2 a+e+\frac{5}{6}\right) u u_{x x}+\left(\frac{5}{4} a+d+1\right) u_{x}^{2}\right]_{x}+O\left(\mu^{2}\right)+O_{L^{\infty}}\left(\mu^{2}\right) .
\end{aligned}
$$

Similarly, one gets

$$
\varepsilon^{2}[v u]_{x}=\varepsilon^{2} \frac{3}{4} u^{2} u_{x}-\varepsilon \mu c^{5}\left(a+\frac{1}{3}\right)\left[u u_{x x}\right]_{x}+O\left(\mu^{2}\right)+O_{L^{\infty}}\left(\mu^{2}\right),
$$

so $(2.9)$ is equivalent to

$$
c u_{t}+\varepsilon v_{t}+c^{2} u_{x}+2 c c_{x} u+2 \varepsilon c u u_{x}+\varepsilon^{2}[v u]_{x}=O_{L^{\infty}}\left(\mu^{2}\right) .
$$

Multiplying by $\frac{1}{c}$, we get

$$
u_{t}+c u_{x}+\frac{3}{2} c_{x} u+\varepsilon \frac{3}{2} u u_{x}-\mu c^{4}\left(a+\frac{1}{3}\right) u_{x x t}=\varepsilon \mu c^{4}\left[-\left(e+a+\frac{1}{2}\right) u u_{x x}-\left(\frac{5}{4} a+d+1\right) u_{x}^{2}\right]_{x}+O_{L^{\infty}}\left(\mu^{2}\right) .
$$

Equating the coefficients of this equation with those of (2.6) shows that the first equation of (2.1) is also satisfied at order $O_{L^{\infty}}\left(\mu^{2}\right)$ if the following relations hold:

$$
a=-\frac{1}{6}, \quad e=-\frac{1}{6}, \quad d=-\frac{19}{48}
$$

and the conditions given in the statement of the proposition on $A, B, E$, and $F$ follows from the expressions of $a, e$ and $d$ given after (2.6).

\subsection{Equations on the surface elevation}

Proceeding exactly as in the proof of Proposition 2.1, one can prove that the family of equations of the surface elevation

$$
\begin{array}{r}
\zeta_{t}+c \zeta_{x}+\frac{1}{2} c_{x} \zeta+\frac{3}{2 c} \varepsilon \zeta \zeta_{x}-\frac{3}{8 c^{3}} \varepsilon^{2} \zeta^{2} \zeta_{x}+\frac{3}{16 c^{5}} \varepsilon^{3} \zeta^{3} \zeta_{x}+\mu\left(\tilde{A} \zeta_{x x x}+B \zeta_{x x t}\right) \\
=\varepsilon \mu \tilde{E} \zeta \zeta_{x x x}+\varepsilon \mu\left(\partial_{x}\left(\frac{\tilde{F}}{2} \zeta\right) \zeta_{x x}+\zeta_{x} \partial_{x}^{2}\left(\frac{\tilde{F}}{2} \zeta\right)\right)
\end{array}
$$

where

$$
\begin{aligned}
\tilde{A} & =A c^{5}-B c^{5}+B c \\
\tilde{E} & =E c^{3}-\frac{3}{2} B c^{3}+\frac{3}{2 c} B \\
\tilde{F} & =F c^{3}-\frac{9}{2} B c^{3}+\frac{9}{2 c} B
\end{aligned}
$$

can be used to construct an approximate solution consistent with the Green-Naghdi equations: 
Proposition 2.2. Let $b \in H^{\infty}(\mathbb{R})$ and $q \in \mathbb{R}$. Assume that

$$
A=q, \quad B=q-\frac{1}{6}, \quad E=-\frac{3}{2} q-\frac{1}{6}, \quad F=-\frac{9}{2} q-\frac{5}{24} .
$$

Then:

- For any family $\wp$ of parameters satisfying $(2.2)$,

- For all $s \geq 0$ large enough and $T>0$,

- For any bounded family $\left(\zeta^{\theta}\right)_{\theta \in \wp} \in C\left(\left[0, \frac{T}{\varepsilon}\right], H^{s}(\mathbb{R})\right)$ solving $(2.10)$,

the family $\left(\zeta^{\theta}, u^{\theta}\right)_{\theta \in \wp}$ with (omitting the index $\theta$ )

$$
u:=\frac{1}{c}\left(\zeta+\frac{c^{2}}{c^{2}+\varepsilon \zeta}\left(-\frac{1}{2} \int_{-\infty}^{x} \frac{c_{x}}{c} \zeta-\frac{\varepsilon}{4 c^{2}} \zeta^{2}-\frac{\varepsilon^{2}}{8 c^{4}} \zeta^{3}+\frac{3 \varepsilon^{3}}{64 c^{6}} \zeta^{4}-\mu \frac{1}{6} c^{3} \zeta_{x t}+\varepsilon \mu c^{2}\left[\frac{1}{6} \zeta \zeta_{x x}+\frac{1}{48} \zeta_{x}^{2}\right]\right)\right)
$$

is $L^{\infty}$-consistent on $\left[0, \frac{T}{\varepsilon}\right]$ with the $G N$ equations (2.1).

Remark 2.3. If we take $b=0$ - i.e. if we consider a flat bottom - then one can recover equation (18) of [8].

Remark 2.4. Choosing $q=\frac{1}{12}, \alpha=\varepsilon$ and $\beta=\mu^{3 / 2}$ equation (2.10) reads after neglecting the $O\left(\mu^{2}\right)$ terms:

$$
\zeta_{t}+c \zeta_{x}+\frac{1}{2} c_{x} \zeta+\frac{3}{2} \varepsilon \zeta \zeta_{x}-\frac{3}{8} \varepsilon^{2} \zeta^{2} \zeta_{x}+\frac{3}{16} \varepsilon^{3} \zeta^{3} \zeta_{x}+\frac{\mu}{12}\left(\zeta_{x x x}-\zeta_{x x t}\right)=-\frac{7}{24} \varepsilon \mu\left(\zeta \zeta_{x x x}+2 \zeta_{x} \zeta_{x x}\right),
$$

it is more advantageous to use this equation (2.12) to study the pattern of wave-breaking for the variable bottom $\mathrm{CH}$ equation (see Sect. 3.2 below).

Proof. As in Proposition 2.1, we make the proof in three steps (we just sketch the proof here since it is similar to the proof of Prop. 2.1).

Step 1. We prove that if $\zeta$ solves (2.10) one gets

$$
\zeta_{t}+c \zeta_{x}+\frac{1}{2} c_{x} u+\frac{3}{2 c} \varepsilon \zeta \zeta_{x}-\frac{3}{8 c^{3}} \varepsilon^{2} \zeta^{2} \zeta_{x}+\frac{3}{16 c^{5}} \varepsilon^{3} \zeta^{3} \zeta_{x}+\mu c^{4} a \zeta_{x x t}=\varepsilon \mu c^{3}\left[e \zeta \zeta_{x x}+d \zeta_{x}^{2}\right]_{x}+O\left(\mu^{2}\right)
$$

with $a=B-A, e=E+\frac{3}{2} A, d=\frac{1}{2}(F+3 A-E)$.

Step 2. We seek $v$ such that if $u:=\frac{1}{c}(\zeta+\varepsilon v)$ and $\zeta$ solves (2.10) then the first equation of (2.1) is satisfied up to a $O_{L^{\infty}}\left(\mu^{2}\right)$ term. Proceeding as in the proof of Proposition 2.1, one can check that a good choice for $v$ is

$$
\left(\frac{c^{2}+\varepsilon \zeta}{c^{2}}\right) \varepsilon v=-\frac{1}{2} \int_{-\infty}^{x} \frac{c_{x}}{c} \zeta-\frac{\varepsilon}{4 c^{2}} \zeta^{2}-\frac{\varepsilon^{2}}{8 c^{4}} \zeta^{3}+\frac{3 \varepsilon^{3}}{64 c^{6}} \zeta^{4}+\mu c^{3} a \zeta_{x t}-\varepsilon \mu c^{2}\left[e \zeta \zeta_{x x}+d \zeta_{x}^{2}\right] .
$$

Step 3. We show here that it is possible to choose the coefficients $A, B, E, F$ such that the second equation of $(2.1)$ is also satisfied up to $O_{L^{\infty}}\left(\mu^{2}\right)$ terms. Replacing $u$ by $\frac{1}{c}(\zeta+\varepsilon v)$ with $v$ given by (2.14), one can check that that this condition is equivalent to

$$
\begin{aligned}
\zeta_{t}+c \zeta_{x}+\frac{1}{2} c_{x} \zeta+\frac{3}{2 c} \varepsilon \zeta \zeta_{x}-\frac{3}{8 c^{3}} \varepsilon^{2} \zeta^{2} \zeta_{x}+\frac{3}{16 c^{5}} \varepsilon^{3} \zeta^{3} \zeta_{x}-\mu c^{4}\left(a+\frac{1}{3}\right) \zeta_{x x t} \\
\quad=\varepsilon \mu c^{3}\left[-\left(e+a-\frac{1}{6}\right) \zeta \zeta_{x x}-\left(\frac{7}{4} a+d+\frac{1}{3}\right) \zeta_{x}^{2}\right]_{x}+O_{L^{\infty}}\left(\mu^{2}\right) .
\end{aligned}
$$


Equating the coefficients of this equation with those of (2.13) shows that the second equation of (2.1) is also satisfied at order $O_{L^{\infty}}\left(\mu^{2}\right)$ if the following relations hold:

$$
a=-\frac{1}{6}, \quad e=-\frac{1}{6}, \quad d=-\frac{1}{48}
$$

and the conditions given in the statement of the proposition on $A, B, E$, and $F$ follows from the expressions of $a, e$ and $d$ given after (2.13).

\subsection{Derivation of the $\mathrm{KdV}$ equation in the long-wave scaling}

In this subsection, attention is given to the regime of slow variations of the bottom topography under the long-wave scaling $\varepsilon=O(\mu)$. We give here a rigorous justification in the meaning of consistency of the variabledepth extensions of the KdV equation (called KdV-top) originally derived in $[12,20,32]$. We consider the values of $\varepsilon, \beta, \alpha$ and $\mu$ satisfying:

$$
\varepsilon=O(\mu), \quad \alpha \beta=O(\varepsilon), \quad \alpha^{2} \beta=O\left(\varepsilon^{2}\right) .
$$

Remark 2.5. Any family of parameters $\theta=(\varepsilon, \beta, \alpha, \mu)$ satisfying (2.14), also satisfies (2.2).

Neglecting the $O\left(\mu^{2}\right)$ terms, one obtains from (2.1) the following Boussinesq system:

$$
\left\{\begin{array}{l}
\zeta_{t}+[h u]_{x}=0 \\
u_{t}+\zeta_{x}+\varepsilon u u_{x}=\frac{\mu}{3} c^{4} u_{x x t}
\end{array}\right.
$$

where we recall that $h=1+\varepsilon \zeta-\beta b^{(\alpha)}$ and $c^{2}=1-\beta b^{(\alpha)}$. The next proposition proves that the KdV-top equation

$$
\zeta_{t}+c \zeta_{x}+\frac{3}{2 c} \varepsilon \zeta \zeta_{x}+\frac{1}{6} \mu c^{5} \zeta_{x x x}+\frac{1}{2} c_{x} \zeta=0,
$$

is $L^{\infty}$-consistent with equations $(2.15)$.

Proposition 2.3. Let $b \in H^{\infty}(\mathbb{R})$. Then:

- For any family $\wp^{\prime}$ of parameters satisfying (2.14),

- For all $s \geq 0$ large enough and $T>0$,

- For any bounded family $\left(\zeta^{\theta}\right)_{\theta \in \wp^{\prime}} \in C\left(\left[0, \frac{T}{\varepsilon}\right], H^{s}(\mathbb{R})\right)$ solving (2.16), the family $\left(\zeta^{\theta}, u^{\theta}\right)_{\theta \in \wp^{\prime}}$ with (omitting the index $\theta$ )

$$
u:=\frac{1}{c}\left(\zeta-\frac{1}{2} \int_{-\infty}^{x} \frac{c_{x}}{c} \zeta-\frac{\varepsilon}{4 c^{2}} \zeta^{2}+\mu \frac{1}{6} c^{4} \zeta_{x x}\right)
$$

is $L^{\infty}$-consistent on $\left[0, \frac{T}{\varepsilon}\right]$ with the equations (2.15).

Remark 2.6. Similarly, one can prove that a family $\left(\zeta^{\theta}, u^{\theta}\right)_{\theta \in \wp^{\prime}}$ with $u^{\theta}$ solution of the KdV-top equation

$$
u_{t}+c u_{x}+\frac{3}{2} \varepsilon u u_{x}+\frac{1}{6} \mu c^{5} u_{x x x}+\frac{3}{2} c_{x} u=0,
$$

and $\zeta^{\theta}$ given by

$$
\zeta:=c u+\frac{1}{2} \int_{-\infty}^{x} c_{x} u+\frac{\varepsilon}{4} u^{2}-\frac{\mu}{6} c^{5} u_{x x},
$$

is $L^{\infty}$-consistent with the equations $(2.15)$.

Proof. We saw in the previous subsection that if $\left(\zeta^{\theta}\right)_{\theta \in \wp}$ is a family of solutions of (2.10), then the family $\left(\zeta^{\theta}, u^{\theta}\right)_{\theta \in \wp}$ with $u^{\theta}$ is given by $(2.11)$ is $L^{\infty}$-consistent with equations $(2.1)$. Since $\theta=(\varepsilon, \beta, \alpha, \mu) \in \wp^{\prime} \subset \wp$ then by taking $q=\frac{1}{6}$, we remark that equations (2.10) and (2.16) are equivalent in the meaning of $L^{\infty}$-consistency and the systems (2.1) and (2.15) are also, so it is clear that $\left(\zeta^{\theta}, u^{\theta}\right)_{\theta \in \wp^{\prime}}$ is $L^{\infty}$-consistent with equations (2.15). 


\section{Mathematical ANAlysis of the VARiable Bottom Models}

\subsection{Well-posedness for the variable bottom $\mathrm{CH}$ equation}

We prove here the well posedness of the general class of equations

$$
\left(1-\mu m \partial_{x}^{2}\right) u_{t}+c u_{x}+k c_{x} u+\sum_{j \in J} \varepsilon^{j} f_{j} u^{j} u_{x}+\mu g u_{x x x}=\varepsilon \mu\left[h_{1} u u_{x x x}+\partial_{x}\left(h_{2} u\right) u_{x x}+u_{x} \partial_{x}^{2}\left(h_{2} u\right)\right]
$$

where $m>0, k \in \mathbb{R}, J$ is a finite subset of $\mathbb{N}^{*}$ and $f_{j}=f_{j}(c), g=g(c), h_{1}=h_{1}(c)$ and $h_{2}=h_{2}(c)$ are smooth functions of $c$. We also recall that $c=\sqrt{1-\beta b^{(\alpha)}}$.

Example 3.1. Taking

$$
\begin{aligned}
& m=-B, \quad k=\frac{3}{2}, \quad J=\{1\}, \quad f_{1}(c)=\frac{3}{2}, \\
& g(c)=A c^{5}-B c^{5}+B c, \quad h_{1}(c)=c^{4} E-\frac{3}{2} B c^{4}+\frac{3}{2} B, \\
& h_{2}(c)=\frac{F c^{4}-\frac{9}{2} B c^{4}+\frac{9}{2} B}{2},
\end{aligned}
$$

equation (3.1) coincides with (1.12).

Example 3.2. Taking

$$
\begin{aligned}
& m=-B, \quad k=\frac{1}{2}, \quad J=\{1,2,3\}, \\
& f_{1}(c)=\frac{3}{2 c}, \quad f_{2}(c)=-\frac{3}{8 c^{3}}, \quad f_{3}(c)=\frac{3}{16 c^{5}}, \\
& g(c)=A c^{5}-B c^{5}+B c, \quad h_{1}(c)=c^{3} E-\frac{3}{2} B c^{3}+\frac{3}{2 c} B, \\
& h_{2}(c)=\frac{F c^{3}-\frac{9}{2} B c^{3}+\frac{9}{2 c} B}{2},
\end{aligned}
$$

equation (3.1) coincides with (2.10).

More precisely, Theorem 3.1 below shows that one can solve the initial-value problem

$$
\mid \begin{aligned}
& \left(1-\mu m \partial_{x}^{2}\right) u_{t}+c u_{x}+k c_{x} u+\sum_{j \in J} \varepsilon^{j} f_{j} u^{j} u_{x}+\mu g u_{x x x}=\varepsilon \mu\left[h_{1} u u_{x x x}+\partial_{x}\left(h_{2} u\right) u_{x x}+u_{x} \partial_{x}^{2}\left(h_{2} u\right)\right] \\
& u_{\mid t=0}=u^{0}
\end{aligned}
$$

on a time scale $O(1 / \varepsilon)$, and under the condition $m>0$. In order to state the result, we need to define the energy space $X^{s}(s \in \mathbb{R})$ as

$$
X^{s+1}(\mathbb{R})=H^{s+1}(\mathbb{R}) \text { endowed with the norm }|f|_{X^{s+1}}^{2}=|f|_{H^{s}}^{2}+\mu m\left|\partial_{x} f\right|_{H^{s}}^{2} .
$$

Theorem 3.1. Let $m>0, s>\frac{3}{2}$ and $b \in H^{\infty}(\mathbb{R})$. Let also $\wp$ be a family of parameters $\theta=(\varepsilon, \beta, \alpha, \mu)$ satisfying (2.2). Then for all $u^{0} \in H^{s+1}(\mathbb{R})$, there exists $T>0$ and a unique family of solutions $\left(u^{\theta}\right)_{\theta \in \wp}$ to (3.2) bounded in $C\left(\left[0, \frac{T}{\varepsilon}\right] ; X^{s+1}(\mathbb{R})\right) \cap C^{1}\left(\left[0, \frac{T}{\varepsilon}\right] ; X^{s}(\mathbb{R})\right)$.

Proof. In this proof, we use the generic notation

$$
C=C\left(\varepsilon, \mu, \alpha, \beta, s,|b|_{H^{\sigma}}\right)
$$


for some $\sigma>s+1 / 2$ large enough. When the constant also depends on $|v|_{X^{s+1}}$, we write $C\left(|v|_{X^{s+1}}\right)$. Note that the dependence on the parameters is assumed to be nondecreasing.

For all $v$ smooth enough, let us define the "linearized" operator $\mathcal{L}(v, \partial)$ as

$$
\mathcal{L}(v, \partial)=\left(1-\mu m \partial_{x}^{2}\right) \partial_{t}+c \partial_{x}+k c_{x}+\varepsilon^{j} f_{j} v^{j} \partial_{x}+\mu g \partial_{x}^{3}-\varepsilon \mu\left[h_{1} v \partial_{x}^{3}+\left(h_{2} v\right)_{x} \partial_{x}^{2}+\left(h_{2} v\right)_{x x} \partial_{x}\right]
$$

for the sake of simplicity we use the convention of summation over related indexes, $\sum_{j \in J} \varepsilon^{j} f_{j} v^{j}=\varepsilon^{j} f_{j} v^{j}$. To construct a solution of (3.1) using an iterative scheme, we have to study the initial-value problem

$$
\left\{\begin{array}{l}
\mathcal{L}(v, \partial) u=\varepsilon f \\
u_{\mid t=0}=u^{0}
\end{array}\right.
$$

If $v$ is smooth enough, it is completely standard to check that for all $s \geq 0, f \in L_{\text {loc }}^{1}\left(\mathbb{R}_{t}^{+} ; H^{s}\left(\mathbb{R}_{x}\right)\right)$ and $u^{0} \in H^{s}(\mathbb{R})$, there exists a unique solution $u \in C\left(\mathbb{R}^{+} ; H^{s+1}(\mathbb{R})\right)$ to $(3.3)$ (recall that $m>0$ ). We thus take for granted the existence of a solution to (3.3) and establish some precise energy estimates on the solution. These energy estimates are given in terms of the $|\cdot|_{X^{s+1}}$ norm introduced above:

$$
|u|_{X^{s+1}}^{2}=|u|_{H^{s}}^{2}+\mu m\left|\partial_{x} u\right|_{H^{s}}^{2} .
$$

Differentiating $\frac{1}{2} \mathrm{e}^{-\varepsilon \lambda t}|u|_{X^{s+1}}^{2}$ with respect to time, one gets using equation (3.3) and integrating by parts,

$$
\begin{aligned}
\frac{1}{2} \mathrm{e}^{\varepsilon \lambda t} \partial_{t}\left(\mathrm{e}^{-\varepsilon \lambda t}|u|_{X^{s+1}}^{2}\right)= & -\frac{\varepsilon \lambda}{2}|u|_{X^{s+1}}^{2}-\left(\Lambda^{s}\left(c \partial_{x} u\right), \Lambda^{s} u\right)-k\left(\Lambda^{s}\left(u \partial_{x} c\right), \Lambda^{s} u\right) \\
& +\varepsilon\left(\Lambda^{s} f, \Lambda^{s} u\right)-\varepsilon^{j}\left(\Lambda^{s}\left(f_{j} v^{j} \partial_{x} u\right), \Lambda^{s} u\right)-\mu\left(\Lambda^{s}\left(g \partial_{x}^{3} u\right), \Lambda^{s} u\right) \\
& -\varepsilon \mu\left(\Lambda^{s}\left(h_{1} v \partial_{x}^{3} u\right), \Lambda^{s} u\right)-\varepsilon \mu\left(\Lambda^{s}\left(\left(h_{2} v\right)_{x} \partial_{x} u\right), \Lambda^{s} \partial_{x} u\right),
\end{aligned}
$$

where $\Lambda=\left(1-\partial_{x}^{2}\right)^{1 / 2}$. Since for all constant coefficient skew-symmetric differential polynomial $P$ (that is, $\left.P^{*}=-P\right)$, and all $h$ smooth enough, one has

$$
\left(\Lambda^{s}(h P u), \Lambda^{s} u\right)=\left(\left[\Lambda^{s}, h\right] P u, \Lambda^{s} u\right)-\frac{1}{2}\left([P, h] \Lambda^{s} u, \Lambda^{s} u\right),
$$

we deduce (applying this identity with $P=\partial_{x}$ and $P=\partial_{x}^{3}$ ),

$$
\begin{aligned}
\frac{1}{2} \mathrm{e}^{\varepsilon \lambda t} \partial_{t}\left(\mathrm{e}^{-\varepsilon \lambda t}|u|_{X^{s+1}}^{2}\right)= & -\frac{\varepsilon \lambda}{2}|u|_{X^{s+1}}^{2}+\varepsilon\left(\Lambda^{s} f, \Lambda^{s} u\right) \\
& -\left(\left[\Lambda^{s}, c\right] \partial_{x} u, \Lambda^{s} u\right)+\frac{1}{2}\left(\left(\partial_{x} c\right) \Lambda^{s} u, \Lambda^{s} u\right)-k\left(\Lambda^{s}\left(u \partial_{x} c\right), \Lambda^{s} u\right) \\
& -\varepsilon^{j}\left(\left[\Lambda^{s}, f_{j} v^{j}\right] \partial_{x} u, \Lambda^{s} u\right)+\frac{\varepsilon^{j}}{2}\left(\partial_{x}\left(f_{j} v^{j}\right) \Lambda^{s} u, \Lambda^{s} u\right) \\
& -\mu\left(\left[\Lambda^{s}, g\right] \partial_{x}^{2} u-\frac{3}{2} g_{x} \Lambda^{s} \partial_{x} u-g_{x x} \Lambda^{s} u, \Lambda^{s} \partial_{x} u\right)-\mu\left(\left[\Lambda^{s}, g_{x}\right] \partial_{x}^{2} u, \Lambda^{s} u\right) \\
& -\varepsilon \mu\left(\left[\Lambda^{s}, h_{1} v\right] \partial_{x}^{2} u-\frac{3}{2}\left(h_{1} v\right)_{x} \Lambda^{s} \partial_{x} u-\left(h_{1} v\right)_{x x} \Lambda^{s} u, \Lambda^{s} \partial_{x} u\right) \\
& -\varepsilon \mu\left(\left[\Lambda^{s},\left(h_{1} v\right)_{x}\right] \partial_{x}^{2} u, \Lambda^{s} u\right)-\varepsilon \mu\left(\Lambda^{s}\left(\left(h_{2} v\right)_{x} \partial_{x} u\right), \Lambda^{s} \partial_{x} u\right) .
\end{aligned}
$$

Note that we also used the identities

$$
\left[\Lambda^{s}, h\right] \partial_{x}^{3} u=\partial_{x}\left(\left[\Lambda^{s}, h\right] \partial_{x}^{2} u\right)-\left[\Lambda^{s}, h_{x}\right] \partial_{x}^{2} u
$$


and

$$
\frac{1}{2}\left(h_{x x x} \Lambda^{s} u, \Lambda^{s} u\right)=-\left(h_{x x} \Lambda^{s} u, \Lambda^{s} u_{x}\right)
$$

The terms involving the velocity $c$ (second line in the r.h.s. of (3.4)) are controlled using the following lemma:

Lemma 3.1. Let $s>3 / 2$ and $(\varepsilon, \beta, \alpha, \mu)$ satisfy (2.2). Then there exists $C>0$ such that

$$
\begin{aligned}
\left|\left(\left[\Lambda^{s}, c\right] \partial_{x} u, \Lambda^{s} u\right)\right| & \leq \varepsilon C|u|_{X^{s+1}}^{2} \\
\left|\left(\left(\partial_{x} c\right) \Lambda^{s} u, \Lambda^{s} u\right)\right| & \leq \varepsilon C|u|_{X^{s+1}}^{2} \\
\left|\left(\Lambda^{s}\left(u \partial_{x} c\right), \Lambda^{s} u\right)\right| & \leq \varepsilon C|u|_{X^{s+1}}^{2} .
\end{aligned}
$$

Proof.

- Estimate of $\left|\left(\left[\Lambda^{s}, c\right] \partial_{x} u, \Lambda^{s} u\right)\right|$. One could control this term by a standard commutator estimates in terms of $\left|c_{x}\right|_{H^{s-1}}$; however, one has $\left|c_{x}\right|_{H^{s-1}}=O(\sqrt{\alpha} \beta)$ and not $O(\alpha \beta)$ as needed. We thus write

$$
\left(\left[\Lambda^{s}, c\right] \partial_{x} u, \Lambda^{s} u\right)=\left(\left(\left[\Lambda^{s}, c\right]-\left\{\Lambda^{s}, c\right\}\right) \partial_{x} u, \Lambda^{s} u\right)+\left(\left\{\Lambda^{s}, c\right\} \partial_{x} u, \Lambda^{s} u\right),
$$

where for all function $F,\left\{\Lambda^{s}, F\right\}$ stands for the Poisson bracket,

$$
\left\{\Lambda^{s}, F\right\}=-s \partial_{x} F \Lambda^{s-2} \partial_{x} .
$$

We can then use the following commutator estimate ([24], Thm. 5): for all $F$ and $U$ smooth enough, one has

$$
\forall s>3 / 2, \quad\left|\left(\left[\Lambda^{s}, F\right]-\left\{\Lambda^{s}, F\right\}\right) U\right|_{2} \leq \operatorname{Cst}\left|\partial_{x}^{2} F\right|_{H^{s}}|U|_{H^{s-2}}^{2} .
$$

Since $\left|\partial_{x}^{2} c\right|_{H^{s}}=O(\alpha \beta)=O(\varepsilon)$, we deduce

$$
\left|\left(\left(\left[\Lambda^{s}, c\right]-\left\{\Lambda^{s}, c\right\}\right) \partial_{x} u, \Lambda^{s} u\right)\right| \leq C \beta \alpha|u|_{X^{s+1}}^{2} \leq C \varepsilon|u|_{X^{s+1}}^{2} .
$$

Moreover

$$
\left|\left(\left\{\Lambda^{s}, c\right\} \partial_{x} u, \Lambda^{s} u\right)\right| \leq\left|-s \partial\left(c^{4}\right) \Lambda^{s-2} \partial_{x}^{2} u\right|_{2}|u|_{X^{s+1}} \leq C\left|\partial\left(c^{4}\right)\right|_{\infty}|u|_{X^{s+1}}^{2} \leq \varepsilon C|u|_{X^{s+1}}^{2},
$$

and thus (3.5) follows easily.

- Estimate of $\left|\left(\left(\partial_{x} c\right) \Lambda^{s} u, \Lambda^{s} u\right)\right|$. Since $|u|_{H^{s}} \leq|u|_{X^{s+1}}$, one has by the Cauchy-Schwarz inequality

$$
\left(\left(\partial_{x} c\right) \Lambda^{s} u, \Lambda^{s} u\right) \leq\left|\partial_{x} c\right|_{\infty}|u|_{X^{s+1}}^{2} ;
$$

therefore, by using the fact that $\left|\partial_{x} c\right|_{\infty} \leq$ Cst $\alpha \beta\left|\partial_{x} b\right|_{\infty}$ and $\alpha \beta=O(\varepsilon)$, one gets easily

$$
\left(\left(\partial_{x} c\right) \Lambda^{s} u, \Lambda^{s} u\right) \leq \varepsilon C|u|_{X^{s+1}}^{2} .
$$

- Estimate of $\left|\left(\Lambda^{s}\left(u \partial_{x} c\right), \Lambda^{s} u\right)\right|$. By Cauchy-Schwarz we get

$$
\left|\left(\Lambda^{s}\left(u \partial_{x} c\right), \Lambda^{s} u\right)\right| \leq\left|u \partial_{x} c\right|_{H^{s}}|u|_{H^{s}}
$$

We have also that

$$
\left|u \partial_{x} c\right|_{H^{s}} \leq\left|\partial_{x} c\right|_{W^{[s]+1, \infty}}|u|_{X^{s+1}}
$$

where $[s]$ is the largest integer smaller or equal to $s$ (this estimate is obvious for $s$ integer and is obtained by interpolation for non integer values of $s$ ). Using this estimate, and the fact that $\beta \alpha=O(\varepsilon)$, it is easy to deduce (3.7). 
For the terms involving the $f_{j}$ (third line in the r.h.s. of (3.4)), we use the controls given by:

Lemma 3.2. Under the assumptions of Theorem 3.1, one has that

$$
\begin{aligned}
\left|\varepsilon^{j}\left(\left[\Lambda^{s}, f_{j} v^{j}\right] \partial_{x} u, \Lambda^{s} u\right)\right| & \leq \varepsilon C\left(|v|_{X^{s+1}}\right)|u|_{X^{s+1}}^{2} \\
\left|\varepsilon^{j}\left(\partial_{x}\left(f_{j} v^{j}\right) \Lambda^{s} u, \Lambda^{s} u\right)\right| & \leq \varepsilon C\left(|v|_{X^{s+1}}\right)|u|_{X^{s+1}}^{2} .
\end{aligned}
$$

Proof.

- Estimate of $\varepsilon^{j}\left(\left[\Lambda^{s}, f_{j} v^{j}\right] \partial_{x} u, \Lambda^{s} u\right)$. By Cauchy-Schwarz we get

$$
\left|\varepsilon^{j}\left(\left[\Lambda^{s}, f_{j} v^{j}\right] \partial_{x} u, \Lambda^{s} u\right)\right| \leq \varepsilon^{j}\left|\left[\Lambda^{s}, f_{j} v^{j}\right] \partial_{x} u\right|_{2}|u|_{X^{s+1}} .
$$

We use here the well-known Calderon-Coifman-Meyer commutator estimate: for all $F$ and $U$ smooth enough, one has

$$
\forall s>3 / 2, \quad\left|\left[\Lambda^{s}, F\right] U\right|_{2} \leq \mathrm{Cst}|F|_{H^{s}}|U|_{H^{s-1}}
$$

using this estimate, it is easy to check that one gets (3.8).

- Estimate of $\varepsilon^{j}\left(\partial_{x}\left(f_{j} v^{j}\right) \Lambda^{s} u, \Lambda^{s} u\right)$. It is clear that

$$
\left|\left(\partial_{x}\left(f_{j} v^{j}\right) \Lambda^{s} u, \Lambda^{s} u\right)\right| \leq\left|\partial_{x}\left(f_{j} v^{j}\right)\right|_{\infty}|u|_{X^{s+1}}^{2} .
$$

Therefore (3.9) follows from the continuous embedding $H^{s} \subset W^{1, \infty}(s>3 / 2)$.

Similarly, the terms involving $g$ (fourth line in the r.h.s. of (3.4)) are controlled using the following lemma:

Lemma 3.3. Under the assumptions of Theorem 3.1, one has that

$$
\left|-\mu\left(\left[\Lambda^{s}, g\right] \partial_{x}^{2} u-\frac{3}{2} g_{x} \Lambda^{s} \partial_{x} u-g_{x x} \Lambda^{s} u, \Lambda^{s} \partial_{x} u\right)-\mu\left(\left[\Lambda^{s}, g_{x}\right] \partial_{x}^{2} u, \Lambda^{s} u\right)\right| \leq \varepsilon C|u|_{X^{s+1}}^{2} .
$$

Proof. Since $|u|_{H^{s}} \leq|u|_{X^{s+1}}$ and $\sqrt{\mu}\left|\partial_{x} u\right|_{H^{s}} \leq \frac{1}{\sqrt{m}}|u|_{X^{s+1}}$, by using the Cauchy-Schwarz inequality and proceeding as for the proofs of Lemmas 3.1 and 3.2 one gets directly (3.10).

Finally to control the terms involving $h_{i}$ (fifth and sixth lines in the r.h.s. of (3.4)), let us state the following lemma:

Lemma 3.4. Under the assumptions of Theorem 3.1, one has that

$$
\begin{gathered}
\left|-\varepsilon \mu\left(\left[\Lambda^{s}, h_{1} v\right] \partial_{x}^{2} u-\frac{3}{2}\left(h_{1} v\right)_{x} \Lambda^{s} \partial_{x} u-\left(h_{1} v\right)_{x x} \Lambda^{s} u, \Lambda^{s} \partial_{x} u\right)-\varepsilon \mu\left(\left[\Lambda^{s},\left(h_{1} v\right)_{x}\right] \partial_{x}^{2} u, \Lambda^{s} u\right)\right| \leq \varepsilon C\left(|v|_{X^{s+1}}\right)|u|_{X^{s+1}}^{2}, \\
\left|-\varepsilon \mu\left(\Lambda^{s}\left(\left(h_{2} v\right)_{x} \partial_{x} u\right), \Lambda^{s} \partial_{x} u\right)\right| \leq \varepsilon C\left(|v|_{X^{s+1}}\right)|u|_{X^{s+1}}^{2} .
\end{gathered}
$$

Proof. We remark first that

$$
\left|\mu\left(\left(h_{1} v\right)_{x x} \Lambda^{s} u, \Lambda^{s} \partial_{x} u\right)\right| \leq\left|\partial_{x}\left(\sqrt{\mu} \partial_{x}\left(h_{1} v\right)\right)\right|_{\infty}|u|_{X^{s+1}}^{2}
$$

since $s-1>\frac{1}{2}$, so by using the imbedding $H^{s-1}(\mathbb{R}) \subset L^{\infty}(\mathbb{R})$ we get

$$
\left|\partial_{x}\left(\sqrt{\mu} \partial_{x}\left(h_{1} v\right)\right)\right|_{\infty} \leq C\left(|v|_{X^{s+1}}\right) .
$$

Proceeding now as in the proof of Lemma 3.3 one gets directly (3.11) and (3.12). 
Gathering the information provided by the above lemmas, we get

$$
\mathrm{e}^{\varepsilon \lambda t} \partial_{t}\left(\mathrm{e}^{-\varepsilon \lambda t}|u|_{X^{s+1}}^{2}\right) \leq \varepsilon\left(C\left(|v|_{X^{s+1}}\right)-\lambda\right)|u|_{X^{s+1}}^{2}+2 \varepsilon|f|_{X^{s+1}}|u|_{X^{s+1}} .
$$

Taking $\lambda=\lambda_{T}$ large enough (how large depending on $\sup _{t \in\left[0, \frac{T}{\varepsilon}\right]} C\left(|v(t)|_{X^{s+1}}\right)$ ) to have the first term of the right hand side negative for all $t \in\left[0, \frac{T}{\varepsilon}\right]$, one deduces

$$
\forall t \in\left[0, \frac{T}{\varepsilon}\right], \quad \partial_{t}\left(\mathrm{e}^{-\varepsilon \lambda_{T} t}|u|_{X^{s+1}}^{2}\right) \leq 2 \varepsilon \mathrm{e}^{-\varepsilon \lambda_{T} t}|f|_{X^{s+1}}|u|_{X^{s+1}}
$$

Integrating this differential inequality yields therefore

$$
\forall t \in\left[0, \frac{T}{\varepsilon}\right], \quad|u(t)|_{X^{s+1}} \leq \mathrm{e}^{\varepsilon \lambda_{T} t}\left|u^{0}\right|_{X^{s+1}}+2 \varepsilon \int_{0}^{t} \mathrm{e}^{\varepsilon \lambda_{T}\left(t-t^{\prime}\right)}\left|u\left(t^{\prime}\right)\right|_{X^{s+1}} \mathrm{~d} t^{\prime} .
$$

Thanks to this energy estimate, we conclude classically (see e.g. [1]) the existence of a

$$
T=T\left(\left|u^{0}\right|_{X^{s+1}}\right)>0,
$$

and of a unique solution $u \in C\left(\left[0, \frac{T}{\varepsilon}\right] ; X^{s+1}(\mathbb{R})\right)$ to $(3.2)$ as a limit of the iterative scheme

$$
u_{0}=u^{0}, \quad \text { and } \quad \forall n \in \mathbb{N}, \quad\left\{\begin{array}{l}
\mathcal{L}\left(u^{n}, \partial\right) u^{n+1}=0 \\
u_{\mid t=0}^{n+1}=u^{0}
\end{array}\right.
$$

Since $u$ solves (3.1), we have $\mathcal{L}(u, \partial) u=0$ and therefore

$$
\left(\Lambda^{s-1}\left(1-\mu m \partial_{x}^{2}\right) \partial_{t} u, \Lambda^{s-1} \partial_{t} u\right)=-\left(\Lambda^{s-1} \mathcal{M}(u, \partial) u, \Lambda^{s-1} \partial_{t} u\right),
$$

with $\mathcal{M}(u, \partial)=\mathcal{L}(u, \partial)-\left(1-\mu m \partial_{x}^{2}\right) \partial_{t}$. Proceeding as above, one gets

$$
\left|\partial_{t} u\right|_{X^{s}} \leq C\left(\left|u^{0}\right|_{X^{s+1}},|u|_{X^{s+1}}\right),
$$

and it follows that the family of solution is also bounded in $C^{1}\left(\left[0, \frac{T}{\varepsilon}\right] ; X^{s}\right)$.

\subsection{Explosion condition for the variable bottom $\mathrm{CH}$ equation}

As in the case of flat bottoms, it is possible to give some information on the blow-up pattern for equation (2.12) for the free surface.

Proposition 3.1. Let $b \in H^{\infty}(\mathbb{R}), \zeta_{0} \in H^{3}(\mathbb{R})$. If the maximal existence time $T_{m}>0$ of the solution of (2.12) with initial profile $\zeta(0, \cdot)=\zeta_{0}$ is finite, the solution $\zeta \in C\left(\left[0, T_{m}\right) ; H^{3}(\mathbb{R})\right) \cap C^{1}\left(\left[0, T_{m}\right) ; H^{2}(\mathbb{R})\right)$ is such that

$$
\sup _{t \in\left[0, T_{m}\right), x \in \mathbb{R}}\{|\zeta(t, x)|\}<\infty
$$

and

$$
\sup _{x \in \mathbb{R}}\left\{\zeta_{x}(t, x)\right\} \uparrow \infty \quad \text { as } \quad t \uparrow T_{m} .
$$

Remark 3.1. It is worth remarking that even though topography effects are introduced in our equation (2.12), wave breaking remains of 'surging' type (i.e. the slope grows to $+\infty$ ) as for flat bottoms. This shows that plunging breakers (i.e. the slope decays to $-\infty$ ) occur for stronger topography variations then those considered in this paper. 
Proof. By using the Theorem 3.1 given $\zeta_{0} \in H^{3}(\mathbb{R})$, the maximal existence time of the solution $\zeta(t)$ to $(2.12)$ with initial data $\zeta(0)=\zeta_{0}$ is finite if and only if $|\zeta(t)|_{H^{3}(\mathbb{R})}$ blows-up in finite time. To complete the proof it is enough to show that:

(i) the solution $\zeta(t)$ given by Theorem 3.1 remains uniformly bounded as long as it is defined; and

(ii) if we can find some $M=M\left(\zeta_{0}\right)>0$ with

$$
\zeta_{x}(t, x) \leq M, \quad x \in \mathbb{R},
$$

as long as the solution is defined, then $|\zeta(t)|_{H^{3}(\mathbb{R})}$ remains bounded on $\left[0, T_{m}\right)$.

Remarking that

$$
\int_{\mathbb{R}}\left[\left(c \zeta_{x}+\frac{1}{2} c_{x} \zeta\right) \zeta\right] \mathrm{d} x=-\int_{\mathbb{R}}\left[(c \zeta)_{x} \zeta-\frac{1}{2} c_{x} \zeta^{2}\right] \mathrm{d} x=-\int_{\mathbb{R}}\left[\left(c \zeta_{x}+\frac{1}{2} c_{x} \zeta\right) \zeta\right] \mathrm{d} x,
$$

we can deduce

$$
\int_{\mathbb{R}}\left[\left(c \zeta_{x}+\frac{1}{2} c_{x} \zeta\right) \zeta\right] \mathrm{d} x=0
$$

We have also that

$$
\int_{\mathbb{R}}\left[\left(\zeta^{i} \zeta_{x}\right) \zeta\right] \mathrm{d} x=0, \quad \forall i \in \mathbb{N}^{*}
$$

Item (i) follows at once from (3.16), (3.17) and the imbedding $H^{1}(\mathbb{R}) \subset L^{\infty}(\mathbb{R})$ since multiplying (2.12) by $\zeta$ and integrating on $\mathbb{R}$ yields

$$
\partial_{t}\left(\int_{\mathbb{R}}\left[\zeta^{2}+\frac{1}{12} \mu \int_{\mathbb{R}} \zeta_{x}^{2}\right] \mathrm{d} x\right)=0 .
$$

To prove item (ii), as in [8] we multiply the equation (2.12) by $\zeta_{x x x x}$ and after performing several integrations by parts we obtains the following identity:

$$
\begin{aligned}
\partial_{t}\left(\int_{\mathbb{R}}\left[\zeta_{x x}^{2}+\frac{1}{12} \mu \int_{\mathbb{R}} \zeta_{x x x}^{2}\right] \mathrm{d} x\right) & =15 \varepsilon \int_{\mathbb{R}} \zeta \zeta_{x x} \zeta_{x x x} \mathrm{~d}-\frac{15}{4} \varepsilon^{2} \int_{\mathbb{R}} \zeta^{2} \zeta_{x x} \zeta_{x x x} \mathrm{~d} x \\
& +\frac{9}{16} \varepsilon^{3} \int_{\mathbb{R}} \zeta_{x}^{5} \mathrm{~d} x+\frac{15}{8} \varepsilon^{3} \int_{\mathbb{R}} \zeta^{3} \zeta_{x x} \zeta_{x x x} \mathrm{~d} x+\frac{7}{4} \mu \varepsilon \int_{\mathbb{R}} \zeta_{x} \zeta_{x x x}^{2} \mathrm{~d} x+I+J
\end{aligned}
$$

where

$$
I=\int_{\mathbb{R}} c \zeta_{x} \zeta_{x x x x} \mathrm{~d} x \quad J=\frac{1}{2} \int_{\mathbb{R}} c_{x} \zeta \zeta_{x x x x} \mathrm{~d} x .
$$

One can use the Cauchy-Schwarz inequality to obtain

$$
\begin{aligned}
I & =-\int_{\mathbb{R}} \zeta_{x}\left(c \zeta_{x}\right)_{x x x}=-\frac{1}{2} \int_{\mathbb{R}} c_{x x x} \zeta_{x}^{2}-\frac{3}{2} \int_{\mathbb{R}} c_{x x} \zeta_{x x} \zeta_{x}-\frac{3}{2} \int_{\mathbb{R}} c_{x} \zeta_{x x x} \zeta_{x} \\
& \leq M_{1}\left(\frac{1}{2}\left|\zeta_{x}\right|_{2}\left|\zeta_{x}\right|_{2}+\frac{3}{2}\left|\zeta_{x x}\right|_{2}\left|\zeta_{x}\right|_{2}+\frac{3}{2}\left|\zeta_{x x x}\right|_{2}\left|\zeta_{x}\right|_{2}\right)
\end{aligned}
$$

and

$$
\begin{aligned}
J & =-\frac{1}{2} \int_{\mathbb{R}} c_{x x} \zeta \zeta_{x x x}-\frac{1}{2} \int_{\mathbb{R}} c_{x} \zeta_{x} \zeta_{x x x} \\
& \leq M_{1}\left(\frac{1}{2}|\zeta|_{2}\left|\zeta_{x x x}\right|_{2}+\frac{1}{2}\left|\zeta_{x}\right|_{2}\left|\zeta_{x x x}\right|_{2}\right)
\end{aligned}
$$


for some $M_{1}=M_{1}\left(|c|_{W^{3, \infty}}\right)>0$. If (3.15) holds, let in accordance with (3.18) $M_{0}>0$ be such that

$$
|\zeta(t, x)| \leq M_{0}, \quad x \in \mathbb{R}
$$

for as long as the solution exists. Using the Cauchy-Schwarz inequality as well as the fact that $\mu \leq 1$, we infer from (3.18), (3.19), (3.20), (3.21) and (3.22) that there exists $C\left(M_{0}, M_{1}, M, \varepsilon, \mu\right)$ such that

$$
\partial_{t} E(t) \leq C\left(M_{0}, M_{1}, M, \varepsilon, \mu\right) E(t),
$$

where

$$
E(t)=\int_{\mathbb{R}}\left[\zeta^{2}+\frac{1}{12} \mu \zeta_{x}^{2}+\zeta_{x x}^{2}+\frac{1}{12} \mu \zeta_{x x x}^{2}\right] \mathrm{d} x .
$$

(Note that we do not give any details for the components of (3.19) other than $I$ and $J$ because these components do not involve any topography term and can therefore be handled exactly as in [8].) An application of Gronwall's inequality enables us to obtain the result.

Our next aim is to show as in the case of flat bottoms that there are solutions to (2.12) that blow-up in finite time as surging breakers, that is, following the pattern given in Proposition 3.1.

Proposition 3.2. Let $b \in H^{\infty}(\mathbb{R})$. If the initial wave profile $\zeta_{0} \in H^{3}(\mathbb{R})$ satisfies

$$
\begin{aligned}
\left|\sup _{x \in \mathbb{R}}\left\{\zeta_{0}(x)\right\}\right|^{2} \geq & \frac{28}{3} C_{0} \mu^{-3 / 4}+\frac{1}{2} \varepsilon C_{0}^{3 / 2} \mu^{-3 / 4}+\frac{1}{4} \varepsilon^{2} C_{0}^{2} \mu^{-3 / 4} \\
& +\frac{7}{3} C_{0} \mu^{-1 / 2}+\frac{8}{3} C_{0}^{1 / 2} C_{1} \mu^{-3 / 4} \varepsilon^{-1}+\frac{4}{3} C_{0}^{1 / 2} C_{1} \mu^{-3 / 4} \varepsilon^{-1},
\end{aligned}
$$

where

$$
C_{0}=\int_{\mathbb{R}}\left[\zeta_{0}^{2}+\frac{1}{12} \mu\left(\zeta_{0}^{\prime}\right)^{2}\right] \mathrm{d} x>0, \quad C_{1}=|c|_{W^{1, \infty}}>0
$$

then wave breaking occurs for the solution of (2.12) in finite time $T=O\left(\frac{1}{\varepsilon}\right)$.

Proof. One can adapt the proof of this proposition in the same way of the proof of the Proposition 6 in [8], and we omit the proof here.

\subsection{Well-posedness for the variable bottom $\mathrm{KdV}$ equation}

We prove now the well-posedness of equations (2.16), (2.17). We consider the following general class of equations

$$
\mid \begin{aligned}
& u_{t}+c u_{x}+k c_{x} u+\varepsilon g u u_{x}+\frac{1}{6} \mu c^{5} u_{x x x}=0 \\
& u_{\left.\right|_{t=0}}=u^{0}
\end{aligned}
$$

where $k \in \mathbb{R}, g=\frac{3}{2}$ for (2.17) and $g=\frac{3}{2 c}$ for (2.16). This class of equations is not included in the family of equations stated in Section 3.1 because $m=0$ (here there is no $\partial_{x}^{2} \partial_{t} u$ term).

Theorem 3.2. Let $s>\frac{3}{2}$ and $b \in H^{\infty}(\mathbb{R})$. Let also $\wp^{\prime}$ be a family of parameters $\theta=(\varepsilon, \beta, \alpha, \mu)$ satisfying (2.14). Assume moreover that

$$
\exists c_{0}>0, \quad \forall \theta \in \wp^{\prime}, \quad c(x)=\sqrt{1-\beta b^{(\alpha)}(x)} \geq c_{0} .
$$

Then for all $u^{0} \in H^{s+1}(\mathbb{R})$, there exists $T>0$ and a unique family of solutions $\left(u^{\theta}\right)_{\theta \in \wp}$ to (3.23) bounded in $C\left(\left[0, \frac{T}{\varepsilon}\right] ; H^{s+1}(\mathbb{R})\right) \cap C^{1}\left(\left[0, \frac{T}{\varepsilon}\right] ; H^{s}(\mathbb{R})\right)$. 
Proof. As in the proof of Theorem 3.1, for all $v$ smooth enough, let us define the "linearized" operator $\mathcal{L}(v, \partial)$ as:

$$
\mathcal{L}(v, \partial)=\partial_{t}+c \partial_{x}+k c_{x}+\varepsilon g v \partial_{x}+\frac{1}{6} \mu c^{5} \partial_{x}^{3}
$$

We define now the initial value problem as:

$$
\left\{\begin{array}{l}
\mathcal{L}(v, \partial) u=\varepsilon f \\
u_{\mid t=0}=u^{0}
\end{array}\right.
$$

Equation (3.24) is a linear equation which can be solved in any interval of time in which the coefficients are defined. We establish some precise energy estimates on the solution. First remark that when $m=0$, the energy norm $|\cdot|_{X^{s}}$ defined in the proof of Theorem 3.1 does not allow to control the term $g u_{x x x}$ (for instance). Indeed, Lemma 3.3 requires $m \neq 0$ to be true. We show that a control of this term is however possible if we use an adequate weight function to defined the energy and use the dispersive properties of the equation. More precisely, inspired by [10] let us define the "energy" norm for all $s \geq 0$ as:

$$
E^{s}(u)^{2}=\left|w \Lambda^{s} u\right|_{2}^{2}
$$

where the weight function $w$ will be determined later. For the moment, we just require that there exists two positive numbers $w_{1}, w_{2}$ such that $\forall x \in \mathbb{R}$

$$
w_{1} \leq w(x) \leq w_{2},
$$

so that $E^{s}(u)$ is uniformly equivalent to the standard $H^{s}$-norm. Differentiating $\frac{1}{2} \mathrm{e}^{-\varepsilon \lambda t} E^{s}(u)^{2}$ with respect to time, one gets using (3.24)

$$
\begin{aligned}
\frac{1}{2} \mathrm{e}^{\varepsilon \lambda t} \partial_{t}\left(\mathrm{e}^{-\varepsilon \lambda t} E^{s}(u)^{2}\right)= & -\frac{\varepsilon \lambda}{2} E^{s}(u)^{2}-\left(\left[\Lambda^{s}, c\right] \partial_{x} u, w \Lambda^{s} u\right)-\left(c \partial \Lambda^{s} u, w \Lambda^{s} u\right) \\
& -k\left(\Lambda^{s}\left(u \partial_{x} c\right), w \Lambda^{s} u\right)-\varepsilon\left(\left[\Lambda^{s}, g v\right] \partial_{x} u, w \Lambda^{s} u\right)-\varepsilon\left(g v \partial \Lambda^{s} u, w \Lambda^{s} u\right) \\
& -\frac{1}{6} \mu\left(\left[\Lambda^{s}, c^{5}\right] \partial_{x}^{3} u, w \Lambda^{s} u\right)-\frac{1}{6} \mu\left(c^{5} \partial^{3} \Lambda^{s} u, w \Lambda^{s} u\right)+\varepsilon\left(\Lambda^{s} f, w \Lambda^{s} u\right) .
\end{aligned}
$$

It is clear, by a simple integration by parts that

$$
\left|\varepsilon\left(g v \partial \Lambda^{s} u, w \Lambda^{s} u\right)\right| \leq \varepsilon C\left(|v|_{W^{1, \infty}},|w|_{W^{1, \infty}}\right) E^{s}(u)^{2} .
$$

Let us now focus on the seventh and eighth terms of the right hand side of the previous identity. In order to get an adequate control of the seventh term, we have to write explicitly the commutator $\left[\Lambda^{s}, c^{5}\right]$ :

$$
\left[\Lambda^{s}, c^{5}\right] \partial_{x}^{3} u=\left\{\Lambda^{s}, c^{5}\right\}_{2} \partial_{x}^{3} u+Q_{1} \partial_{x}^{3} u,
$$

where $\{\cdot, \cdot\}_{2}$ stands for the second order Poisson brackets,

$$
\left\{\Lambda^{s}, c^{5}\right\}_{2}=-s \partial_{x}\left(c^{5}\right) \Lambda^{s-2} \partial_{x}+\frac{1}{2}\left[s \partial_{x}^{2}\left(c^{5}\right) \Lambda^{s-2}-s(s-2) \partial_{x}^{2}\left(c^{5}\right) \Lambda^{s-4} \partial_{x}^{2}\right]
$$

and $Q_{1}$ is an operator of order $s-3$ that can be controlled by the general commutator estimates of (see [24]). We thus get

$$
\left|\left(Q_{1} \partial_{x}^{3} u, w \Lambda^{s} u\right)\right| \leq C \varepsilon E^{s}(u)^{2} .
$$


We now use the identity $\Lambda^{2}=1-\partial_{x}^{2}$ and the fact that $\alpha \beta=O(\varepsilon)$, to get, as in (3.25),

$$
\left|\left(\left[s \partial_{x}^{2}\left(c^{5}\right) \Lambda^{s-2}-s(s-2) \partial_{x}^{2}\left(c^{5}\right) \Lambda^{s-4} \partial_{x}^{2}\right] \partial_{x}^{3} u, w \Lambda^{s} u\right)\right| \leq \varepsilon C\left(|w|_{W^{1, \infty}}\right) E^{s}(u)^{2} .
$$

This leads to the expression

$$
\frac{1}{6} \mu\left(\left[\Lambda^{s}, c^{5}\right] \partial_{x}^{3} u, w \Lambda^{s} u\right)=\frac{s}{6} \mu\left(\partial c^{5} \Lambda^{s} \partial^{2} u, w \Lambda^{s} u\right)+Q_{2},
$$

where $\left|Q_{2}\right| \leq \varepsilon C\left(|w|_{W^{1, \infty}}\right) E^{s}(u)^{2}$. Remarking now that

$$
\frac{s}{6} \mu\left(\partial c^{5} \Lambda^{s} \partial^{2} u, w \Lambda^{s} u\right)=-\frac{s}{6} \mu\left(\partial\left(\partial\left(c^{5}\right) w\right) \Lambda^{s} \partial u, \Lambda^{s} u\right)-\frac{s}{6} \mu\left(\partial\left(c^{5}\right) w,\left(\Lambda^{s} \partial u\right)^{2}\right) .
$$

The control of the eighth term comes in the same way:

$$
\frac{1}{6} \mu\left(c^{5} \partial^{3} \Lambda^{s} u, w \Lambda^{s} u\right)=-\frac{1}{12} \mu\left(\partial^{3}\left(c^{5} w\right) \Lambda^{s} u, \Lambda^{s} u\right)-\frac{1}{4} \mu\left(\partial^{2}\left(w c^{5}\right) \Lambda^{s} \partial u, \Lambda^{s} u\right)-\frac{1}{4} \mu\left(\partial\left(w c^{5}\right) \Lambda^{s} u, \Lambda^{s} \partial^{2} u\right)
$$

similarly:

$$
-\frac{1}{4} \mu\left(\partial\left(w c^{5}\right) \Lambda^{s} u, \Lambda^{s} \partial^{2} u\right)=\frac{1}{4} \mu\left(\partial^{2}\left(c^{5} w\right) \Lambda^{s} \partial u, \Lambda^{s} u\right)+\frac{1}{4} \mu\left(\partial\left(c^{5} w\right),\left(\Lambda^{s} \partial u\right)^{2}\right) .
$$

We choice here $w$ so that

$$
-\frac{s}{6} \mu\left(\partial\left(c^{5}\right) w,\left(\Lambda^{s} \partial u\right)^{2}\right)+\frac{1}{4} \mu\left(\partial\left(c^{5} w\right),\left(\Lambda^{s} \partial u\right)^{2}\right)=0
$$

therefore if one take $w=c^{5\left(\frac{2 s}{3}-1\right)}$ we get easily (3.26). Finally, one has

$$
\begin{aligned}
\frac{1}{6} \mu\left(\left[\Lambda^{s}, c^{5}\right] \partial_{x}^{3} u, w \Lambda^{s} u\right)+\frac{1}{6} \mu\left(c^{5} \partial^{3} \Lambda^{s} u, w \Lambda^{s} u\right)= & Q_{2}-\frac{s}{6} \mu\left(\partial\left(\partial\left(c^{5}\right) w\right) \Lambda^{s} \partial u, \Lambda^{s} u\right)-\frac{1}{12} \mu\left(\partial^{3}\left(c^{5} w\right) \Lambda^{s} u, \Lambda^{s} u\right) \\
& -\frac{1}{4} \mu\left(\partial^{2}\left(c^{5} w\right) \Lambda^{s} \partial u, \Lambda^{s} u\right)+\frac{1}{4} \mu\left(\partial^{2}\left(c^{5} w\right) \Lambda^{s} \partial u, \Lambda^{s} u\right),
\end{aligned}
$$

using again the fact that $\alpha \beta=O(\varepsilon)$ one can deduce

$$
\mathrm{e}^{\varepsilon \lambda t} \partial_{t}\left(\mathrm{e}^{-\varepsilon \lambda t} E^{s}(u)^{2}\right) \leq \varepsilon\left(C\left(E^{s}(v)\right)-\lambda\right) E^{s}(u)^{2}+4 \varepsilon E^{s}(f) E^{s}(u) .
$$

This inequality, together with end of the proof of Theorem 3.1, easily yields the result.

\section{Rigorous JustificAtion of THE VARIABLE BOtTOM EQUATIONS}

\subsection{Rigorous justification of the variable bottom $\mathrm{CH}$ equation}

We restrict here our attention to parameters $\varepsilon, \beta, \alpha$ and $\mu$ linked by the relations

$$
\varepsilon=O(\sqrt{\mu}), \beta \alpha=O(\varepsilon), \beta \alpha=O\left(\mu^{2}\right) .
$$

These conditions are stronger than (2.2), and this allows us to control the secular effects that prevented us from proving an $H^{s}$-consistency (and a fortiori a full justification) for the variable bottom equations of Section 2. The notion of $H^{s}$-consistency is defined below: 
Definition 4.1. Let $\wp_{1}$ be a family of parameters $\theta=(\varepsilon, \beta, \alpha, \mu)$ satisfying (4.1). A family $\left(\zeta^{\theta}, u^{\theta}\right)_{\theta \in \wp_{1}}$ is $H^{s}$-consistent of order $s \geq 0$ and on $\left[0, \frac{T}{\varepsilon}\right]$ with the GN equations (2.1), if for all $\theta \in \wp_{1}$ (and denoting $\left.h^{\theta}=1+\varepsilon \zeta^{\theta}-\beta b^{(\alpha)}\right)$,

$$
\left\{\begin{array}{l}
\zeta_{t}^{\theta}+\left[h^{\theta} u\right]_{x}=\mu^{2} r_{1}^{\theta} \\
u_{t}^{\theta}+\zeta_{x}^{\theta}+\varepsilon u^{\theta} u_{x}^{\theta}=\frac{\mu}{3} \frac{1}{h^{\theta}}\left[\left(h^{\theta}\right)^{3}\left(u_{x t}^{\theta}+\varepsilon u^{\theta} u_{x x}^{\theta}-\varepsilon\left(u_{x}^{\theta}\right)^{2}\right)\right]_{x}+\mu^{2} r_{2}^{\theta}
\end{array}\right.
$$

with $\left(r_{1}^{\theta}, r_{2}^{\theta}\right)_{\theta \in \wp_{1}}$ bounded in $L^{\infty}\left(\left[0, \frac{T}{\varepsilon}\right], H^{s}(\mathbb{R})^{2}\right)$.

Remark 4.1. Since $H^{s}(\mathbb{R})$ is continuously embedded in $L^{\infty}(\mathbb{R})$ for $s>1 / 2$, the $H^{s}$-consistency implies the $L^{\infty}$-consistency when $s>1 / 2$.

Proposition 4.1. Let $b \in H^{\infty}(\mathbb{R})$ and $p \in \mathbb{R}$. Assume that

$$
A=p, \quad B=p-\frac{1}{6}, \quad E=-\frac{3}{2} p-\frac{1}{6}, \quad F=-\frac{9}{2} p-\frac{23}{24}
$$

Then:

- For any family $\wp_{1}$ of parameters satisfying (4.1),

- For all $s \geq 0$ large enough and $T>0$,

- For any bounded family $\left(u^{\theta}\right)_{\theta \in \wp_{1}} \in C\left(\left[0, \frac{T}{\varepsilon}\right], H^{s}(\mathbb{R})\right)$ solving (1.12), the family $\left(\zeta^{\theta}, u^{\theta}\right)_{\theta \in \wp_{1}}$ with (omitting the index $\theta$ )

$$
\zeta:=c u+\frac{\varepsilon}{4} u^{2}+\frac{\mu}{6} c^{4} u_{x t}-\varepsilon \mu c^{4}\left[\frac{1}{6} u u_{x x}+\frac{5}{48} u_{x}^{2}\right]
$$

is $H^{s}$-consistent on $\left[0, \frac{T}{\varepsilon}\right]$ with the GN equations (2.1).

Proof. This is clear by using the same arguments of the proof of Proposition 2.1 if we remark that now the term $c_{x} u$ (responsible of the secular growth effects) is of order $O\left(\mu^{2}\right)$ in $L^{\infty}\left(\left[0, \frac{T}{\varepsilon}\right], H^{s}(\mathbb{R})\right)$. Therefore this quantity is not required in the identity (2.7) that defines $v$. In the proof of Proposition 2.1 there is therefore no $c_{x} u$ term and the $O_{L^{\infty}}\left(\mu^{2}\right)$ terms in Proposition 2.1 are now of order $O\left(\mu^{2}\right)$.

In Proposition 4.1, we constructed a family $\left(u^{\theta}, \zeta^{\theta}\right)$ consistent with the Green-Naghdi equations in the sense of Definition 4.1. A consequence of the following theorem is a stronger result: this family provides a good approximation of the exact solutions $\left(\underline{u}^{\theta}, \underline{\zeta}^{\theta}\right)$ of the Green-Naghdi equations with same initial data in the sense that $\left(\underline{u}^{\theta}, \underline{\zeta}^{\theta}\right)=\left(u^{\theta}, \zeta^{\theta}\right)+O\left(\mu^{2} t\right)$ for times $O(1 / \varepsilon)$.

Theorem 4.1. Let $b \in H^{\infty}(\mathbb{R}), s \geq 0$ and $\wp_{1}$ be a family of parameters satisfying $(4.1)$ with $\beta=O(\varepsilon)$. Let also $A, B, E$ and $F$ be as in Proposition 4.1. If $B<0$ then there exists $D>0, P>D$ and $T>0$ such that for all $u_{0}^{\theta} \in H^{s+P}(\mathbb{R})$ :

- There is a unique family $\left(u^{\theta}, \zeta^{\theta}\right)_{\theta \in \wp_{1}} \in C\left(\left[0, \frac{T}{\varepsilon}\right] ; H^{s+P}(\mathbb{R}) \times H^{s+P-2}\right)$ given by the resolution of (1.12) with initial condition $u_{0}^{\theta}$ and formula $(4.2)$;

- There is a unique family $\left(\underline{u}^{\theta}, \underline{\zeta}^{\theta}\right)_{\theta \in \wp_{1}} \in C\left(\left[0, \frac{T}{\varepsilon}\right] ; H^{s+D}(\mathbb{R})^{2}\right)$ solving the Green-Naghdi equations (2.1) with initial condition $\left(u_{0}^{\theta}, \zeta_{\left.\right|_{t=0}}^{\theta} \overline{)}\right.$.

Moreover, one has for all $\theta \in \wp_{1}$,

$$
\forall t \in\left[0, \frac{T}{\varepsilon}\right], \quad\left|\underline{u}^{\theta}-u^{\theta}\right|_{L^{\infty}([0, t] \times \mathbb{R})}+\left|\underline{\zeta}^{\theta}-\zeta^{\theta}\right|_{L^{\infty}([0, t] \times \mathbb{R})} \leq \text { Cst } \mu^{2} t .
$$

Remark 4.2. It is known (see [2]) that the Green-Naghdi equations give, under the scaling $(1.9)$ with $\beta=O(\varepsilon)$ a correct approximation of the exact solutions of the full water waves equations (with a precision $O\left(\mu^{2} t\right)$ and 
over a time scale $O(1 / \varepsilon)$ ). It follows that the unidirectional approximation discussed above approximates the solution of the water waves equations with the same accuracy.

Remark 4.3. We used the unidirectional equations derived on the velocity as the basis for the approximation justified in the Theorem 4.1. One could of course use instead the unidirectional approximation (2.10) derived on the surface elevation.

Proof. The first point of the theorem is a direct consequence of Theorem 3.1. Thanks to Proposition 4.1, we know that $\left(u^{\theta}, \zeta^{\theta}\right)_{\theta \in \wp_{1}}$ is $H^{s}$-consistent with the Green-Naghdi equations (2.1), so that the second point of the theorem and the error estimate follow at once from the well-posedness and stability of the Green-Naghdi equations under the present scaling (see Thm. 4.10 of [3]).

\subsection{Rigorous justification of the variable bottom KdV-top equation}

In this subsection the parameters $\varepsilon, \beta, \alpha$ and $\mu$ are assumed to satisfy

$$
\varepsilon=O(\mu), \beta \alpha=O\left(\varepsilon^{2}\right) .
$$

We give first a proposition regarding the $H^{s}$-consistency result for the KdV-top equation:

$$
\zeta_{t}+c \zeta_{x}+\frac{3}{2 c} \varepsilon \zeta \zeta_{x}+\frac{1}{6} \mu c^{5} \zeta_{x x x}+\frac{1}{2} c_{x} \zeta=0 .
$$

Proposition 4.2. Let $b \in H^{\infty}(\mathbb{R})$. Then:

- For any family $\wp_{1}^{\prime}$ of parameters satisfying (4.3),

- For all $s \geq 0$ large enough and $T>0$,

- For any bounded family $\left(\zeta^{\theta}\right)_{\theta \in \wp_{1}^{\prime}} \in C\left(\left[0, \frac{T}{\varepsilon}\right], H^{s}(\mathbb{R})\right)$ solving (4.4),

the family $\left(\zeta^{\theta}, u^{\theta}\right)_{\theta \in \wp^{\prime}}$ with (omitting the index $\theta$ )

$$
u:=\frac{1}{c}\left(\zeta-\frac{\varepsilon}{4 c^{2}} \zeta^{2}+\mu \frac{1}{6} c^{4} \zeta_{x x}\right)
$$

is $H^{s}$-consistent on $\left[0, \frac{T}{\varepsilon}\right]$ with the equations $(2.1)$.

Remark 4.4. Similarly, one can prove that the solution of the following KdV-top equations

$$
u_{t}+c u_{x}+\frac{3}{2} \varepsilon u u_{x}+\frac{1}{6} \mu c^{5} u_{x x x}+\frac{3}{2} c_{x} u=0,
$$

on the velocity, with

$$
\zeta:=c u+\frac{\varepsilon}{4} u^{2}-\frac{\mu}{6} c^{5} u_{x x}
$$

is $H^{s}$-consistent with the equations Green-Naghdi equations (2.1).

Proof. One can adapt the proof of Proposition 2.3 in the same way as we adapted the proof of Proposition 2.1 to establish Proposition 4.1 (we also use the fact that if a family is consistent with the Boussinesq equations (2.15), it is also consistent with the Green-Naghdi equations (2.1) under the present scaling).

The following theorem deals with the rigorous justification of the KdV variable bottom equation:

Theorem 4.2. Let $b \in H^{\infty}(\mathbb{R}), s \geq 0$ and $\wp_{1}^{\prime}$ be a family of parameters satisfying (4.3) with $\beta=O(\varepsilon)$. If $B<0$ then there exist $D>0, P>D$ and $T>0$ such that for all $\zeta_{0}^{\theta} \in H^{s+P}(\mathbb{R})$ :

- There is a unique family $\left(\zeta^{\theta}, u^{\theta}\right)_{\theta \in \wp_{1}^{\prime}} \in C\left(\left[0, \frac{T}{\varepsilon}\right] ; H^{s+P-2}(\mathbb{R}) \times H^{s+P}\right)$ given by the resolution of (4.4) with initial condition $\zeta_{0}^{\theta}$ and formula (4.5);

- There is a unique family $\left(\underline{\zeta}^{\theta}, \underline{u}^{\theta}\right)_{\theta \in \wp_{1}^{\prime}} \in C\left(\left[0, \frac{T}{\varepsilon}\right] ; H^{s+D}(\mathbb{R})^{2}\right)$ solving the Green-Naghdi equations (2.1) with initial condition $\left(\zeta_{0}^{\theta}, u_{\mid t=0}^{\theta}\right)$. 
Moreover, one has for all $\theta \in \wp_{1}^{\prime}$,

$$
\forall t \in\left[0, \frac{T}{\varepsilon}\right], \quad\left|\underline{u}^{\theta}-u^{\theta}\right|_{L^{\infty}([0, t] \times \mathbb{R})}+\left|\underline{\zeta}^{\theta}-\zeta^{\theta}\right|_{L^{\infty}([0, t] \times \mathbb{R})} \leq \operatorname{Cst} \varepsilon^{2} t .
$$

Remark 4.5. We used the unidirectional equation derived for the free surface elevation as the basis for the approximation justified in the Theorem 4.2. One could of course use instead the unidirectional approximation (4.6) derived on velocity.

Remark 4.6. It is known (see $[6,16,31]$ ) that in the $\mathrm{KdV}$ scaling $\varepsilon=O(\mu)$ and for flat bottoms, any initial condition for the Boussinesq (or Green-Naghdi) equations splits up into two counter propagating waves, each of which evolving according to a KdV equation. It is possible to choose the initial condition in order to cancel the left going wave (for instance); one thus gets a purely rightgoing wave. In the more nonlinear regime $\varepsilon=O(\sqrt{\mu})$ investigated in [8] and in the present paper, the initial condition under consideration is of this kind in the sense that it leads to a right going wave (up to small corrector terms). It is not clear however that any general initial condition splits up into two counter propagating waves, each of which evolving according to an equation of the form (1.12). Indeed, the nonlinear terms are more important in this regime and the nonlinear interaction of the counter propagating components may grow secularly (see [22] for a description of this phenomenon). The presence of topography terms makes such a general decoupling even more difficult to establish since they also induce secular terms in the backscattered component (this is the reason why we can only prove $L^{\infty}$-consistency in Prop. 2.1). If such a decoupling holds, it will therefore be with a weaker precision than in the KdV, flat bottom, regime.

Proof. The first point of the theorem is a direct consequence of Theorem 3.2. Thanks to Proposition 4.2, we know that $\left(u^{\theta}, \zeta^{\theta}\right)_{\theta \in \wp_{1}^{\prime}}$ is $H^{s}$-consistent with the Green-Naghdi equations (2.1), so that the second point follows as in Theorem 4.1 .

Acknowledgements. The author is grateful to David Lannes for encouragement and many helpful discussions. This work was supported by the ANR grant MathOcean ANR-08-BLAN-0301-01.

\section{REFERENCES}

[1] S. Alinhac and P. Gérard, Opérateurs pseudo-différentiels et théorème de Nash-Moser. EDP Sciences, Les Ulis, France (1991).

[2] B. Alvarez-Samaniego and D. Lannes, Large time existence for $3 D$ water-waves and asymptotics. Invent. Math. 171 (2008) 485-541.

[3] B. Alvarez-Samaniego and D. Lannes, A Nash-Moser theorem for singular evolution equations. Application to the Serre and Green-Naghdi equations. Indiana Univ. Math. J. 57 (2008) 97-131.

[4] T.B. Benjamin, J.L. Bona and J.J. Mahony, Model equations for long waves in nonlinear dispersive systems. Phil. Trans. Roy. Soc. London A 227 (1972) 47-78.

[5] J.L. Bona, M. Chen and J.-C. Saut, Boussinesq equations and other systems for small-amplitude long waves in nonlinear dispersive media. I. Derivation and linear theory. J. Nonlinear Sci. 12 (2002) 283-318.

[6] J.L. Bona, T. Colin and D. Lannes, Long wave approximations for water waves. Arch. Ration. Mech. Anal. 178 (2005) $373-410$.

[7] R. Camassa and D. Holm, An integrable shallow water equation with peaked solitons. Phys. Rev. Lett. 71 (1993) 1661-1664.

[8] A. Constantin and D. Lannes, The hydrodynamical relevance of the Camassa-Holm and Degasperis-Processi equations. Arch. Ration. Mech. Anal. 192 (2009) 165-186.

[9] W. Craig, An existence theory for water waves and the Boussinesq and the Korteweg-de Vries scaling limits. Commun. Partial Differ. Equations 10 (1985) 787-1003.

[10] W. Craig, T. Kappeler and W. Strauss, Gain of regularity for equations of KdV type. Ann. Institut Henri Poincaré, Anal. non linéaire 9 (1992) 147-186.

[11] A. Degasperis and M. Procesi, Asymptotic integrability, in Symmetry and Perturbation Theory, A. Degasperis and G. Gaeta Eds., World Scientific, Singapore (1999) 23-37.

[12] M.W. Dingemans, Water waves propogation over uneven bottoms. Part 2. Advanced Series on ocean Engineering 13. World Scientific, Singapore (1997).

[13] P.G. Drazin and R.S. Johnson, Solitons: an introduction. Cambridge University Press, Cambridge, UK (1992). 
[14] A.E. Green and P.M. Naghdi, A derivation of equations for wave propagation in water of variable depth. J. Fluid Mech. 78 (1976) 237-246.

[15] R. Grimshaw and S.R. Pudjaprasetya, Hamiltonian formulation for solitary waves propagating on a variable background. J. Engrg. Math. 36 (1999) 89-98.

[16] T. Iguchi, A long wave approximation for capillary-gravity waves and the Kawahara equation. Bull. Inst. Math. Acad. Sin. (N.S.) 2 (2007) $179-220$.

[17] R.S. Johnson, A modern introduction to the mathematical theory of water waves. Cambridge University Press, Cambridge, UK (1997).

[18] R.S. Johnson, Camassa-Holm, Korteweg-de Vries and related models for water waves. J. Fluid Mech. 457 (2002) 63-82.

[19] R.S. Johnson, On the development of a solitary wave moving over an uneven bottom. Proc. Cambridge Philos. Soc. 73 (1973) 183-203.

[20] J.J. Kirby, Nonlinear ocean surface waves. Center for Applied Coastal research, University of Delaware, USA (2004).

[21] D.J. Korteweg and G. de Vries, On the change of form of long waves advancing in a rectangular canal and on a new type of long stationary waves. Phil. Mag. 39 (1895) 422.

[22] D. Lannes, Secular growth estimates for hyperbolic systems. J. Differ. Equ. 190 (2003) 466-503.

[23] D. Lannes, Well-posedness of the water waves equations. J. Amer. Math. Soc. 18 (2005) 605-654.

[24] D. Lannes, Sharp estimates for pseudo-differential operators with symbols of limited smoothness and commutators. J. Funct. Anal. 232 (2006) 495-539.

[25] D. Lannes and P. Bonneton, Derivation of asymptotic two-dimensional time-dependent equations for surface water wave propagation. Phys. Fluids 21 (2009).

[26] J.W. Miles, On the Korteweg-de Vries equation for a gradually varying channel. J. Fluid Mech. 91 (1979) 181-190.

[27] V.I. Nalimov, [The Cauchy-Poisson problem]. Dinamika Splošn. Sredy Vyp. 18 Dinamika Zidkost. so Svobod. Granicami 254 (1974) 104-210 (in Russian).

[28] D.H. Peregrine, Calculations of the development of an undular bore. J. Fluid Mech. 25 (1966) 321-330.

[29] S.R. Pudjaprasetya and E. van Groesen, Unidirectional waves over slowly varying bottom. II. Quasi-homogeneous approximation of distorting waves. Wave Motion 23 (1996) 23-38.

[30] S.R. Pudjaprasetya, E. van Groesen and E. Soewono, The splitting of solitary waves running over a shallower water. Wave Motion 29 (1999) 375-389.

[31] G. Schneider and C. Wayne, The long-wave limit for the water wave problem I. The case of zero surface tension. Commun. Pure Appl. Math. 53 (2000) 1475-1535.

[32] I.A. Svendsen, A direct derivation of the KDV equation for waves on a beach, and discussion of it's implications. Prog. Rept. 39, ISVA, Tech. Univ. of Denmark (1976) 9-14.

[33] E. van Groesen and S.R. Pudjaprasetya, Uni-directional waves over slowly varying bottom. I. Derivation of a KdV-type of equation. Wave Motion 18 (1993) 345-370.

[34] S. Wu, Well-posedness in Sobolev spaces of the full water wave problem in 2-D. Invent. Math. 130 (1997) 39-72.

[35] S. Wu, Well-posedness in Sobolev spaces of the full water wave problem in 3-D. J. Amer. Math. Soc. 12 (1999) $445-495$.

[36] S.B. Yoon and P.L.-F. Liu, A note on Hamiltonian for long water waves in varying depth. Wave Motion 20 (1994) 359-370.

[37] H. Yosihara, Gravity waves on the free surface of an incompressible perfect fluid of finite depth. Publ. Res. Inst. Math. Sci. 18 (1982) 49-96. 